\title{
Inheritance of Some Important Characters to Improve Sweet Melon (Cucumis melo L.) Fruits
}

\author{
Abou kamer, M. E*, Mona. M. Yousry ${ }^{\star \star}$, A. K. Hatem, A.M. El-Gamal** \\ "Horticultural Research Institute, Agricultural Research Center, Egypt. \\ "Plant Production Department, Faculty of Agriculture (Saba Basha), Alex. University
}

\begin{abstract}
The present investigation was carried out during three years of 2012, 2013 and 2014. These experiments were done at Sabaheya Horticultural Research Station, Alexandria, and Fowa area, Kafer El- Sheikh, Egypt. Type of gene action, correlation coefficient and path analysis among all combinations of some important characteristics of sweet melon were studied. A 5X5 half-diallel cross was performed among five pure lines of sweet melon. Additive gene effects were found to be significant for plant length, number of branches / plant, flowering date, maturity date and flesh thickness indicating that the additive gene action played the main role in the inheritance of these traits. The evaluated characteristics of fruit netting, fruit shape index, total soluble solids \% and moisture content exhibited insignificant values for the additive gene action. The dominant gene effect was found to be significant for plant length, number of branches per plant, maturity date, fruit netting degree, Total soluble solids $\%$ and moisture content indicating the importance of dominant gene effect in the inheritance of this characters. Total yield per plant, phenotypically, correlated with plant length, average fruit number and average fruit weight. Hence, a lot of attention for such relationships in the improvement program of such characters of sweet melon through selection.
\end{abstract}

Key words: Cucumis melo, gene action, correlation coefficients, path analysis

\section{INTRODUCTION}

Sweet melon (Cucumis melo L.) is one of the most important economic species of the family Cucurbitaceae. Among the different parts of a melon plant, fruits have the highest diversity in size, form, external ornamentation, and internal and external color (Kirkbride, 1993). Further, Kirkbride (1993) and Goldman (2002) reported that, fruits as short as $4 \mathrm{~cm}$ long (C. melo, L. var agrestis) and as long as $200 \mathrm{~cm}$ (C. melo, L. var. flexuosus ) and attaining weights between $50 \mathrm{~g}$ and more than $15 \mathrm{~kg}$. Plant breeding programs are aim to improve the characteristics of plant so that they become more desirable agronomically and economically higher yield and improved quality. High yield, early maturity and uniform fruit shape and size, as well as, excellent quality, are important objectives for melon breeding programs (Zalapa et al. 2006). Several researchers had match attention with the family Cucurbitaceae to study the influence of gene action; such as, Zalapa et al. (2006), Feyzian et al. (2009), Pornsuriya et al. (2009) and Abu Arak (2013), they all declared that the additive genes effects were the key regulator factor for most melon traits. They, also, showed that all three types of epistatic effects were significant for fruit width (additive $\mathrm{x}$ additive, additive $\mathrm{x}$ dominance and dominance $\mathrm{x}$ dominance) effected in the inheritance of melon traits.

The objectives of the present study were: (1) to generate genetic information such as, the nature of gene action (additive, dominant, and epistasis gene action) controlling the studied characters and (2) calculating the correlation coefficient $(r)$ for different pairs of some important characters of sweet melon to be used in the improvement programs of melon. 


\section{MATERIALS AND METHODS}

\section{Experimental materials}

The genetic material were consisted of five parental lines obtained from the breeding program of the project of improvement the Cucurbitaceae vegetables, Horticultural Research Institute. Five genotypes are (line kooz Assal $\left(P_{1}\right)$, Line Matrouh $\left(P_{2}\right)$, line orange $\left(P_{3}\right)$, line green $\left(P_{4}\right)$ and line Ideal $\left.\left(P_{5}\right)\right)$. A $5 \times 5$ half diallel cross was performed, in the green house at the first of February in 2012, among the five lines of sweet melon, to get $10 \mathrm{~F} 1$ hybrid combinations. At the first of august 2012, the F1 of each of the ten hybrids was selfed and backcrossed to both parents to get $10 \mathrm{~F} 2$ and 20 backcross populations which were sown on $15^{\text {th }}$ of March, 2013 and $20^{\text {th }}$ of March, 2014.

\section{Field experiments and the experimental design}

The seeds of the 5 parents, $10 \mathrm{~F} 1^{\prime} \mathrm{s}, 10 \mathrm{~F} 2^{\prime} \mathrm{s}$ and 20 backcrosses generations, as well as, the seeds of the commercial cultivar "Gallia" were sown for the evaluation on $15^{\text {th }}$ and $20^{\text {th }}$ of March 2013 and 2014; respectively, in Fowa area, Kafer El- Sheikh Governorate, Egypt. A randomized complete blocks design with three replicates was used. Each plot consisted of three rows; each row was $4 \mathrm{~m}$ long and 1 meter wide having an area $12 \mathrm{~m}^{2}$ for each plot.

\section{Statistical analysis and estimation of genetic parameters}

The recorded data for the six populations; i.e., $\mathrm{P}_{1}, \mathrm{P}_{2}, \mathrm{~F}_{1}, \mathrm{~F}_{2}, \mathrm{BC}_{1}$ and $\mathrm{BC}_{2}$ for each cross were, statistically, analyzed and the combined analysis over two seasons were done as outlined by Allard (1960). Types of gene action were calculated using relationships given by Hayman (1958) and Gamble (1962). Simple correlation coefficient $(r)$ was calculated for different pairs of the studied characters as shown by Dospekhove (1984).

\section{RESULTS AND DISCUSSION}

Data of Table (1 and 2) revealed that there were significant genotypic differences among the tested populations for all the studied characters. This result indicating that the evaluated populations differed in their genetic potential with respect to these traits. The environmental factor (year effects) showed significant and highly significant effects on plant height, number of branches per plant, flowering date, maturity date, fruit flesh thickness, netting degree and moisture content. The results indicated that there were fluctuations in the environmental conditions from year to another throughout both experiments of this investigation affecting these characters. The interaction between the genetically and environmental factors (genotype $X$ year) had pronounced effects for the two traits flowering date and T.S.S \%, suggested that the relative performance of the evaluated population was essentially the same, when grown under individual environments, as illustrated by Anne et al. (2011).

The mean values of the vegetative characters are shown in Table (3). Results of plant height trait showed that most of the $F_{1}$ 's crosses had the highest mean values. The hybrid $2 \times 4$ and $3 \times 4$ led to the tallest plant but the shortest plant was given by $\mathrm{BC}_{1} \mathrm{P}_{1}$ in the hybrid $2 \times 4$. According to number of branches per plant trait. Results showed, generally, that the $F_{1}$ 's plants recorded high values where it ranged between (4- 4.66). The crosses $1 \times 3,1 \times 5$ 
and $3 \times 5$ recorded the lowest mean values for flowering date characters, so these genotypes might be elected for producing early fruits. On the other hand, the genotypes $P_{1}, 2 \times 4,2 \times 5$ and Gallia 1 might be elected for producing late fruit, where they all gave values ranged between 40 to51 days from planting to flowering stage. For maturity date the results showed that the hybrids $1 \times 3,1 \times 2$, $1 \times 5,3 \times 5,2 \times 3$ and $4 \times 5$ recorded the lowest value for the days to maturity.

Table(1):Combined analysis of variance for the studied vegetative characters; flowering date, maturity date and yield and its components of 5 parents, $10 F_{1}, 10$ F2,10 BC1P1 and 10 BC1P2 sweet melon crosses (over two years of 2013 and 2014)

\begin{tabular}{ccccccccc}
\hline S.O.V. & D.F. $\begin{array}{c}\text { Plant height } \\
(\mathrm{cm})\end{array}$ & $\begin{array}{c}\text { No. of } \\
\text { branches } \\
\text { per plant }\end{array}$ & $\begin{array}{c}\text { Flowering } \\
\text { date (day) }\end{array}$ & $\begin{array}{c}\text { Maturity } \\
\text { date (day) }\end{array}$ & $\begin{array}{c}\text { Fruits } \\
\text { No. per } \\
\text { plant }\end{array}$ & $\begin{array}{c}\text { Average } \\
\text { fruit } \\
\text { weight } \\
(\mathrm{kg})\end{array}$ & $\begin{array}{c}\text { Total fruit } \\
\text { yield per } \\
\text { plant }(\mathrm{kg})\end{array}$ \\
\hline Blocks & 2 & $3728.38^{*}$ & $1.65^{*}$ & 3.15 & 7.95 & 0.72 & 0.096 & 0.311 \\
Genotypes & 44 & $3254.67^{* *}$ & $1.15^{* *}$ & $82.61^{* *}$ & $354.79^{* *}$ & $0.70^{* *}$ & $0.232^{* *}$ & $1.122^{* *}$ \\
Years & 1 & $4670.84^{*}$ & $2.70^{*}$ & $31.33^{* *}$ & $6.69^{* *}$ & 0.18 & 0.183 & 4.206 \\
GxY & 44 & 671.35 & 0.40 & $11.00^{* *}$ & 79.01 & 0.41 & 0.074 & 0.240 \\
Error & 178 & 1037.64 & 0.19 & 3.46 & 2.42 & 0.42 & 0.091 & 0.248
\end{tabular}

*, ** Significant and highly significant at the 0.05 and 0.01 level of probability, respectively.

Table(2):Combined analysis of variance for the studied fruit characteristics of 5 parents, $10 \mathrm{~F}_{1}, 10 \mathrm{F2}, 10 \mathrm{BC} 1 \mathrm{P} 1$ and $10 \mathrm{BC} 1 \mathrm{P} 2$ sweet melon crosses (over two years of 2013 and 2014)

\begin{tabular}{lcccccc}
\hline \multicolumn{1}{c}{ S.O.V. } & D.F. & $\begin{array}{c}\text { Fruit flesh } \\
\text { Thickness } \\
(\%)\end{array}$ & $\begin{array}{c}\text { Fruit shape } \\
\text { index }\end{array}$ & $\begin{array}{c}\text { Fruit netting } \\
\text { degree }\end{array}$ & $\begin{array}{c}\text { T.S.S } \\
(\%)\end{array}$ & $\begin{array}{c}\text { Moisture } \\
\text { Content (\%) }\end{array}$ \\
\hline Blocks & 2 & $184.058^{* *}$ & 0.025 & $4.959^{* *}$ & 2.718 & $12.873^{*}$ \\
Genotypes & 44 & $39.161^{* *}$ & $0.085^{* *}$ & $3.168^{* *}$ & $7.607^{* *}$ & $7.547^{* *}$ \\
Seasons & 1 & $273.330^{* *}$ & 0.019 & 0.003 & $11.408^{*}$ & $25.330^{*}$ \\
Gx S & 44 & 19.052 & 0.008 & 0.700 & $2.841^{*}$ & 3.841 \\
Error & 178 & 21.612 & 0.010 & 0.985 & 1.845 & 3.890
\end{tabular}

${ }^{*},{ }^{* *} \overline{\text { Significant and highly significant at the } 0.05 \text { and } 0.01 \text { level of probability, respectively. }}$

The mean performances for yield and yield component character are presented in Table (4). The highest average for the fruit number per plant recorded by the $P_{2}$ (line green) followed by the $F_{1}$ plants for the hybrids $1 \times 2$, $1 \times 3,1 \times 4,2 \times 4,2 \times 5,3 \times 4$ and $3 \times 5$. Results indicated that the lowest fruit number was obtained by the parent $\mathrm{P}_{1}$ (line kooz Assal). For average of fruit weight per plant character results clearly showed that the genotype $B C_{1} P_{1}$ in cross $1 \times 4$ 
recorded the highest fruit weight value followed by the genotype $\mathrm{BC}_{1} \mathrm{P} 2$ in the cross $2 \times 3$. The highest $F_{1}$ values for the fruit weight character were recorded by the hybrids $1 \times 3,1 \times 4$ and $2 \times 4$. The highest recorded value for the total fruit yield per plant trait was obtained by the genotype $\mathrm{BC}_{1} \mathrm{P}_{1}$ in the cross $1 \times 4$, while the highest $F_{1}$ values were given by the hybrids $1 \times 2,1 \times 5,2 \times 4,2 \times 5,3 \times 4$ and $3 \times 5$.

Mean performances of sweet melon fruit characteristics are presented in Table (5).The mean values of fruit flesh thickness showed that the $F_{1}$ plants ( cross $3 \times 5$ ) gave the highest value, followed by the commercial cultivar "Gallia 1" compared with other evaluated genotypes. Regarding the fruit shape index character, the parent $P_{1}$ (line kooz Assal) seemed to have an oblong fruit shape where it gave the highest value; the $F_{1}$ crosses seemed to be round where values ranged between 0.90 and 1.27. The lowest value was given by "Gallia 1" $\mathrm{cv}$., where it recorded 0.90 for this trait. Most of the $F_{1}$ crosses getting the highest values in addition to the check cultivar "Gallia 1", but the lowest values were recorded with $\mathrm{BC}_{1} \mathrm{P}_{2}$ in the cross $2 \times 4$.

The total soluble solids character (T.S.S) for the $\mathrm{F} 1$ crosses ranged from $12.83 \%$ (the hybrid $1 \times 4$ ) to $16.86 \%$ for the cross $1 \times 5$; while the lowest mean value was recorded by the $P_{1}$ (line kooz Assal). The cultivar "Gallia 1" recorded moderate percentage in this respect. The parent $P_{1}$ (line kooz Assal) gave the highest percentage value $(93.77 \%)$ for the fruit moisture content trait; while all the $F_{1}$ crosses ranged from $89.54 \%$ (the hybrid $2 \times 3$ ) to $92.27 \%$ for the hybrid $4 \times 5$.The P3(line orange) recorded the lowest value $(83.72 \%)$ for the fruit moisture content trait.

Chamnan et al. (2006) in Thai Slicing melon reported that, $F_{1}$ generation had the best performance considered from fruit number per plant, followed by that of $\mathrm{BC}_{2}$, whereas, the poorest performance was $\mathrm{P}_{1}$. Zalapa et al. (2006) in melon found that, for most traits, $F_{1}$ generation means were higher than the mid-parent value, and at $A R$ the mean of the $F_{1}$ surpassed the mean of the high parent for fruit number per plant, fruit weight per plant and days to anthesis. Also, they found that the $F_{1}$ generation was intermediate to parental lines for primary branch number at both AR (5.7) and HCK (5.6), and performed equal to/or better than both parents for fruit number per plant (5.9, AR and 1.7, HCK), fruit weight per plant $(6.2 \mathrm{~kg}, \mathrm{AR}$ and $2.4 \mathrm{~kg}, \mathrm{HCK})$ and average weight per fruit $(1.1 \mathrm{~kg}, \mathrm{AR}$ and $1.5 \mathrm{~kg}, \mathrm{HCK}) . \mathrm{BC}_{1} \mathrm{P}_{1}$ and $\mathrm{BC}_{1} \mathrm{P}_{2}$ progeny resembled their respective recurrent parent with respect to growth habit and fruiting characteristics, and F2 individuals varied dramatically for the yield-related characteristics examined. 
Table (3): Mean performances of 5 parents, $10 F_{1}, 10 \mathrm{~F} 2,10 \mathrm{BC} 1 \mathrm{P} 1,10$ BC1P2 sweet melon crosses and a check cultivar for the studied vegetative characters, flowering date and maturity date (over two years of 2013 and 2014)

\begin{tabular}{|c|c|c|c|c|c|c|c|c|}
\hline genotypes & $\begin{array}{l}\text { Plant } \\
\text { height } \\
(\mathrm{cm})\end{array}$ & $\begin{array}{l}\text { No. of } \\
\text { branches } \\
\text { per plant }\end{array}$ & $\begin{array}{c}\text { Flowering } \\
\text { Date } \\
\text { (day) }\end{array}$ & $\begin{array}{l}\text { Maturity } \\
\text { date } \\
\text { (day) }\end{array}$ & $\begin{array}{l}\text { Plant } \\
\text { height } \\
\text { (cm) }\end{array}$ & $\begin{array}{l}\text { No. of } \\
\text { branches } \\
\text { per plant }\end{array}$ & $\begin{array}{l}\text { Flowering } \\
\text { date (day) }\end{array}$ & $\begin{array}{l}\text { Maturity } \\
\text { date } \\
\text { (day) }\end{array}$ \\
\hline \multicolumn{5}{|c|}{ Cross $1(1 \times 2)$} & \multicolumn{4}{|c|}{ Cross $6(2 \times 4)$} \\
\hline $\mathrm{P}_{1}$ & $225.50^{\mathrm{bc} *}$ & $4.66^{\mathrm{a}}$ & $43.00^{b}$ & $79.00^{e}$ & $236.60^{b}$ & $3.83^{\mathrm{a}}$ & $35.50^{g}$ & $79.50^{c}$ \\
\hline $\mathrm{P}_{2}$ & $236.60^{\mathrm{ab}}$ & $3.83^{b}$ & $35.50^{g}$ & $79.50^{d}$ & $230.20^{b c}$ & $4.33^{a}$ & $37.50^{f}$ & $79.50^{c}$ \\
\hline $\mathrm{F}_{1}$ & $263.50^{a}$ & $4.66^{a}$ & $36.00^{\dagger}$ & $70.00^{\mathrm{g}}$ & $279.50^{a}$ & $4.00^{a}$ & $40.00^{d}$ & $78.50^{\mathrm{e}}$ \\
\hline $\mathrm{F}_{2}$ & $198.80^{C}$ & $3.83^{b}$ & $39.00^{\mathrm{e}}$ & $75.00^{f}$ & $192.20^{c}$ & $4.50^{\mathrm{a}}$ & $39.50^{\mathrm{e}}$ & $79.00^{d}$ \\
\hline $\mathrm{BC}_{1}$ & $224.60^{\mathrm{bc}}$ & $4.16^{\mathrm{ab}}$ & $41.00^{d}$ & $86.50^{\mathrm{b}}$ & $191.30^{c}$ & $4.00^{\mathrm{a}}$ & $40.50^{c}$ & $78.00^{f}$ \\
\hline $\mathrm{BC}_{2}$ & $223.50^{b c}$ & $4.50^{\mathrm{ab}}$ & $41.50^{C}$ & $81.00^{C}$ & $207.00^{\mathrm{bc}}$ & $3.83^{a}$ & $44.50^{\mathrm{b}}$ & $91.00^{\mathrm{b}}$ \\
\hline Gallia1 & $209.00^{b c}$ & $3.83^{\mathrm{b}}$ & $51.00^{\mathrm{a}}$ & $92.00^{\mathrm{a}}$ & $209.00^{b c}$ & $3.83^{\mathrm{a}}$ & $51.00^{\mathrm{a}}$ & $92.00^{\mathrm{a}}$ \\
\hline \multicolumn{5}{|c|}{ Cross $2(1 \times 3)$} & \multicolumn{4}{|c|}{ Cross $7(2 \times 5)$} \\
\hline $\mathrm{P}_{1}$ & $225.50^{\mathrm{ab}}$ & $4.66^{\mathrm{ab}}$ & $43.00^{b}$ & $79.00^{c}$ & $236.60^{\text {ab }}$ & $3.83^{\mathrm{ab}}$ & $35.50^{g}$ & $79.50^{e}$ \\
\hline$P_{2}$ & $234.10^{\mathrm{ab}}$ & $4.33^{\mathrm{ab}}$ & $38.50^{d}$ & $76.00^{\mathrm{e}}$ & $214.00^{b c}$ & $3.50^{\mathrm{ab}}$ & $37.00^{\mathrm{e}}$ & $80.00^{d}$ \\
\hline$F_{1}$ & $270.10^{a}$ & $4.66^{\mathrm{ab}}$ & $34.50^{g}$ & $69.50^{g}$ & $261.30^{a}$ & $4.00^{\mathrm{a}}$ & $40.00^{d}$ & $80.50^{c}$ \\
\hline $\mathrm{F}_{2}$ & $212.50^{b}$ & $3.83^{\mathrm{b}}$ & $37.50^{e}$ & $81.50^{\mathrm{b}}$ & $186.30^{c}$ & $3.66^{\mathrm{ab}}$ & $36.00^{\dagger}$ & $79.00^{f}$ \\
\hline $\mathrm{BC}_{1}$ & $227.8^{0 a b}$ & $4.33^{\mathrm{ab}}$ & $40.50^{c}$ & $77.50^{d}$ & $216.30^{\mathrm{bc}}$ & $3.66^{\mathrm{ab}}$ & $48.50^{b}$ & $87.00^{\mathrm{b}}$ \\
\hline $\mathrm{BC}_{2}$ & $219.30^{b}$ & $4.83^{\mathrm{a}}$ & $37.00^{\dagger}$ & $73.00^{\dagger}$ & $233.00^{\mathrm{ab}}$ & $3.16^{b}$ & $41.50^{\mathrm{C}}$ & $76.00^{\mathrm{g}}$ \\
\hline Gallia1 & $209.00^{b}$ & $3.83^{\mathrm{b}}$ & $51.00^{\mathrm{a}}$ & $92.00^{\mathrm{a}}$ & $209.00^{b c}$ & $3.83^{\mathrm{ab}}$ & $51.00^{\mathrm{a}}$ & $92.00^{\mathrm{a}}$ \\
\hline \multicolumn{5}{|c|}{ Cross $3(1 \times 4)$} & \multicolumn{4}{|c|}{ Cross $8(3 \times 4)$} \\
\hline $\mathrm{P}_{1}$ & $225.50^{\mathrm{ab}}$ & $4.66^{\mathrm{a}}$ & $43.00^{c}$ & $79.00^{d}$ & $234.20^{b}$ & $4.33^{\mathrm{a}}$ & $38.50^{f}$ & $76.00^{9}$ \\
\hline $\mathrm{P}_{2}$ & $230.20^{\mathrm{ab}}$ & $4.33^{\mathrm{a}}$ & $37.50^{\mathrm{e}}$ & $79.50^{\mathrm{C}}$ & $230.10^{\mathrm{b}}$ & $4.33^{a}$ & $37.50^{9}$ & $79.50^{f}$ \\
\hline $\mathrm{F}_{1}$ & $259.60^{a}$ & $4.00^{\mathrm{a}}$ & $35.50^{\dagger}$ & $78.50^{\mathrm{e}}$ & $279.50^{\mathrm{a}}$ & $4.16^{a}$ & $39.50^{d}$ & $88.00^{\mathrm{e}}$ \\
\hline $\mathrm{F}_{2}$ & $207.60^{b}$ & $3.00^{\mathrm{b}}$ & $35.00^{\mathrm{g}}$ & $75.50^{\mathrm{g}}$ & $207.00^{\mathrm{b}}$ & $4.16^{a}$ & $39.00^{\mathrm{e}}$ & $91.50^{\mathrm{C}}$ \\
\hline $\mathrm{BC}_{1}$ & $246.50^{\mathrm{ab}}$ & $4.83^{\mathrm{a}}$ & $43.50^{b}$ & $77.00^{\dagger}$ & $220.80^{\mathrm{b}}$ & $3.83^{\mathrm{a}}$ & $41.00^{\mathrm{C}}$ & $89.00^{d}$ \\
\hline $\mathrm{BC}_{2}$ & $218.30^{\mathrm{ab}}$ & $4.83^{\mathrm{a}}$ & $40.00^{d}$ & $84.00^{\mathrm{b}}$ & $222.50^{\mathrm{b}}$ & $3.66^{a}$ & $44.50^{\mathrm{b}}$ & $93.50^{\mathrm{a}}$ \\
\hline Gallia1 & $209.00^{b}$ & $3.83^{\mathrm{ab}}$ & $51.00^{\mathrm{a}}$ & $92.00^{\mathrm{a}}$ & $209.00^{\mathrm{b}}$ & $3.83^{\mathrm{a}}$ & $51.00^{\mathrm{a}}$ & $92.00^{\mathrm{b}}$ \\
\hline \multicolumn{5}{|c|}{ Cross $4(1 \times 5)$} & \multicolumn{4}{|c|}{ Cross $9(3 \times 5)$} \\
\hline $\mathrm{P}_{1}$ & $225.50^{\mathrm{abc}}$ & $4.66^{\mathrm{a}}$ & $43.00^{b}$ & $79.00^{\dagger}$ & $237.50^{\mathrm{ab}}$ & $4.33^{\mathrm{ab}}$ & $38.50^{\mathrm{e}}$ & $76.00^{\dagger}$ \\
\hline $\mathrm{P}_{2}$ & $214.00^{\mathrm{abc}}$ & $3.50^{\mathrm{b}}$ & $37.00^{\dagger}$ & $80.00^{\mathrm{e}}$ & $214.00^{b}$ & $3.50^{\mathrm{c}}$ & $37.00^{\dagger}$ & $80.00^{\mathrm{e}}$ \\
\hline $\mathrm{F}_{1}$ & $251.30^{\mathrm{a}}$ & $4.33^{\mathrm{ab}}$ & $36.00^{\mathrm{g}}$ & $74.50^{g}$ & $259.80^{a}$ & $4.66^{\mathrm{a}}$ & $34.00^{9}$ & $74.50^{g}$ \\
\hline $\mathrm{F}_{2}$ & $199.30^{c}$ & $4.33^{\mathrm{ab}}$ & $37.50^{\mathrm{e}}$ & $94.00^{\mathrm{a}}$ & $217.30^{\mathrm{b}}$ & $3.66^{\mathrm{bc}}$ & $41.00^{d}$ & $82.00^{d}$ \\
\hline $\mathrm{BC}_{1}$ & $213.20^{\mathrm{abc}}$ & $4.50^{\mathrm{ab}}$ & $40.50^{c}$ & $92.50^{\mathrm{C}}$ & $217.20^{b}$ & $4.33^{\mathrm{ab}}$ & $42.00^{c}$ & $84.00^{\mathrm{C}}$ \\
\hline $\mathrm{BC}_{2}$ & $241.00^{\mathrm{ab}}$ & $4.00^{\mathrm{ab}}$ & $38.50^{d}$ & $93.50^{\mathrm{b}}$ & $207.60^{\mathrm{b}}$ & $4.16^{\mathrm{abc}}$ & $48.50^{b}$ & $89.00^{b}$ \\
\hline Gallia1 & $209.00^{\text {bc }}$ & $3.83^{\mathrm{ab}}$ & $51.00^{\mathrm{a}}$ & $92.00^{d}$ & $209.00^{b}$ & $3.83^{\mathrm{bc}}$ & $51.00 \mathrm{a}$ & $92.00^{\mathrm{a}}$ \\
\hline \multicolumn{5}{|c|}{ Cross $5(2 \times 3)$} & \multicolumn{4}{|c|}{ Cross $10(4 \times 5)$} \\
\hline $\mathrm{P}_{1}$ & $236.60^{a}$ & $3.83^{\mathrm{a}}$ & $35.50^{d}$ & $79.50^{\mathrm{e}}$ & $230.20^{\mathrm{ab}}$ & $4.33^{\mathrm{a}}$ & $37.50^{f}$ & $79.50^{d}$ \\
\hline $\mathrm{P}_{2}$ & $234.20^{\mathrm{a}}$ & $4.33^{\mathrm{a}}$ & $38.50^{c}$ & $76.00^{\dagger}$ & $214.00^{\mathrm{b}}$ & $3.50^{\mathrm{bc}}$ & $37.00^{\mathrm{g}}$ & $80.00^{c}$ \\
\hline$F_{1}$ & $239.30^{\mathrm{a}}$ & $4.16^{\mathrm{a}}$ & $36.00^{d}$ & $75.00^{\mathrm{g}}$ & $256.30^{\mathrm{a}}$ & $4.16^{\mathrm{ab}}$ & $39.00^{d}$ & $75.50^{f}$ \\
\hline $\mathrm{F}_{2}$ & $216.50^{a}$ & $4.16^{a}$ & $39.00^{C}$ & $96.50^{\mathrm{a}}$ & $213.20^{b}$ & $3.16^{c}$ & $38.50^{\mathrm{e}}$ & $79.00^{e}$ \\
\hline $\mathrm{BC}_{1}$ & $232.00^{\mathrm{a}}$ & $4.50^{\mathrm{a}}$ & $41.50^{b}$ & $81.00^{d}$ & $209.00^{b}$ & $4.00^{\mathrm{ab}}$ & $43.50^{\mathrm{b}}$ & $84.50^{\mathrm{b}}$ \\
\hline $\mathrm{BC}_{2}$ & $249.30^{a}$ & $3.83^{\mathrm{a}}$ & $41.50^{\mathrm{b}}$ & $82.50^{C}$ & $222.80^{\mathrm{ab}}$ & $4.00^{\mathrm{ab}}$ & $43.00^{c}$ & $79.00^{\mathrm{e}}$ \\
\hline Gallia1 & $209.00^{\mathrm{b}}$ & $3.83^{a}$ & $51.00^{\mathrm{a}}$ & $92.00^{\mathrm{b}}$ & $209.00^{\mathrm{b}}$ & $3.83^{a b c}$ & $51.00^{\mathrm{a}}$ & $92.00^{\mathrm{a}}$ \\
\hline
\end{tabular}


Table (4): Mean performances of 5 parents, $10 F_{1}, 10 F_{2}, 10 B C_{1} P_{1}, 10$ $\mathrm{BC}_{1} \mathrm{P}_{2}$ sweet melon crosses and a check cultivar for the studied yield and yield components (over two years of 2013 and 2014)

\begin{tabular}{|c|c|c|c|c|c|c|}
\hline genotypes & $\begin{array}{l}\text { Fruits No. } \\
\text { per Plant }\end{array}$ & $\begin{array}{l}\text { Average fruit } \\
\text { weight }(\mathrm{kg})\end{array}$ & $\begin{array}{l}\text { Total fruit yield } \\
\text { per plant }(\mathrm{kg})\end{array}$ & $\begin{array}{c}\text { Fruits No. per } \\
\text { Plant }\end{array}$ & $\begin{array}{c}\text { Average fruit } \\
\text { weight }(\mathrm{kg})\end{array}$ & $\begin{array}{l}\text { Total fruit yield } \\
\text { per plant }(\mathrm{kg})\end{array}$ \\
\hline \multicolumn{4}{|c|}{ Cross 1 $(1 \times 2)$} & \multicolumn{3}{|c|}{ Cross $6(2 \times 4)$} \\
\hline$P_{1}$ & $1.83^{\mathrm{c}^{*}}$ & $1.180^{\mathrm{a}}$ & $2.140^{\mathrm{ab}}$ & $2.83^{\mathrm{a}}$ & $0.765^{a b c}$ & $2.150^{\mathrm{b}}$ \\
\hline$P_{2}$ & $2.83^{a b}$ & $0.765^{\mathrm{c}}$ & $2.150^{\mathrm{ab}}$ & $3.17^{\mathrm{a}}$ & $0.739^{b c}$ & $2.307^{\mathrm{b}}$ \\
\hline $\mathrm{F}_{1}$ & $3.00^{a}$ & $0.831^{b c}$ & $2.473^{a}$ & $3.00^{\mathrm{a}}$ & $1.029^{a b}$ & $2.854^{a}$ \\
\hline $\mathrm{F}_{2}$ & $2.16^{\mathrm{bc}}$ & $0.755^{\mathrm{c}}$ & $1.370^{\mathrm{C}}$ & $2.00^{b}$ & $1.111^{\mathrm{a}}$ & $1.825^{b}$ \\
\hline $\mathrm{BC}_{1}$ & $2.83^{\mathrm{ab}}$ & $0.648^{c}$ & $1.751^{\mathrm{bc}}$ & $2.50^{\mathrm{ab}}$ & $0.762^{a b c}$ & $1.893^{b}$ \\
\hline $\mathrm{BC}_{2}$ & $2.33^{a b c}$ & $1.051^{\mathrm{ab}}$ & $2.320^{\mathrm{a}}$ & $1.83^{\mathrm{b}}$ & $0.651^{\mathrm{c}}$ & $1.161^{\mathrm{c}}$ \\
\hline Gallia1 & $2.33^{a b c}$ & $0.872^{b c}$ & $2.008^{a b}$ & $2.33^{a b}$ & $0.871^{a b c}$ & $2.008^{b}$ \\
\hline \multicolumn{4}{|c|}{ Cross $2(1 \times 3)$} & \multicolumn{3}{|c|}{ Cross $7(2 \times 5)$} \\
\hline$P_{1}$ & $1.83^{b}$ & $1.225^{\mathrm{a}}$ & $2.148^{a b}$ & $2.83^{\mathrm{ab}}$ & $0.765^{a b}$ & $2.150^{\mathrm{a}}$ \\
\hline $\mathrm{P}_{2}$ & $2.50^{\mathrm{ab}}$ & $0.983^{a}$ & $2.171^{\mathrm{ab}}$ & $2.50^{b}$ & $0.728^{b}$ & $1.820^{\mathrm{a}}$ \\
\hline $\mathrm{F}_{1}$ & $2.16^{\mathrm{ab}}$ & $1.100^{\mathrm{a}}$ & $2.205^{a b}$ & $3.33^{a}$ & $0.718^{b}$ & $2.280^{\mathrm{a}}$ \\
\hline $\mathrm{F}_{2}$ & $2.33^{a b}$ & $0.910^{a}$ & $1.918^{\mathrm{b}}$ & $2.33^{b}$ & $1.015^{\mathrm{a}}$ & $2.280^{\mathrm{a}}$ \\
\hline $\mathrm{BC}_{1}$ & $3.00^{\mathrm{a}}$ & $1.085^{\mathrm{a}}$ & $2.735^{\mathrm{a}}$ & $2.50^{\mathrm{b}}$ & $0.645^{\mathrm{b}}$ & $1.610^{\mathrm{a}}$ \\
\hline $\mathrm{BC}_{2}$ & $2.33^{\mathrm{ab}}$ & $1.105^{\mathrm{a}}$ & $2.455^{a b}$ & $2.50^{b}$ & $0.853^{a b}$ & $2.080^{\mathrm{a}}$ \\
\hline Gallia1 & $2.33^{\mathrm{ab}}$ & $0.871^{\mathrm{a}}$ & $2.008^{\mathrm{b}}$ & $2.33^{b}$ & $0.872^{\mathrm{ab}}$ & $2.020^{\mathrm{a}}$ \\
\hline \multicolumn{4}{|c|}{ Cross 3(1x 4) } & \multicolumn{3}{|c|}{ Cross $8(3 \times 4)$} \\
\hline$P_{1}$ & $1.83^{c}$ & $1.353^{\mathrm{ab}}$ & $2.148^{b c}$ & $2.50^{b}$ & $0.983^{a}$ & $2.171^{\mathrm{ab}}$ \\
\hline $\mathrm{P}_{2}$ & $3.16^{\mathrm{a}}$ & $0.739^{c}$ & $2.307^{b c}$ & $3.16^{\mathrm{a}}$ & $0.739^{a}$ & $2.307^{a b}$ \\
\hline $\mathrm{F}_{1}$ & $2.66^{\mathrm{ab}}$ & $1.093^{b c}$ & $2.526^{a b}$ & $2.66^{a b}$ & $0.974^{a}$ & $2.546^{\mathrm{a}}$ \\
\hline $\mathrm{F}_{2}$ & $2.33^{b c}$ & $0.793^{c}$ & $1.626^{c}$ & $2.33^{b}$ & $0.846^{a}$ & $2.085^{\mathrm{ab}}$ \\
\hline $\mathrm{BC}_{1}$ & $2.16^{\mathrm{bc}}$ & $1.602^{\mathrm{a}}$ & $3.029^{a}$ & $2.50^{b}$ & $0.710^{\mathrm{a}}$ & $1.744^{\mathrm{b}}$ \\
\hline $\mathrm{BC}_{2}$ & $2.66^{\mathrm{ab}}$ & $0.794^{\mathrm{c}}$ & $2.159^{b c}$ & $2.16^{b}$ & $0.876^{a}$ & $1.780^{\mathrm{b}}$ \\
\hline Gallia1 & $2.33^{\mathrm{bc}}$ & $0.871^{b c}$ & $2.023^{b c}$ & $2.33^{b}$ & $0.871^{a}$ & $2.008^{a b}$ \\
\hline \multicolumn{4}{|c|}{ Cross $4(1 \times 5)$} & \multicolumn{3}{|c|}{ Cross $9(3 \times 5)$} \\
\hline$P_{1}$ & $1.83^{b}$ & $1.180^{a}$ & $2.148^{b}$ & $2.5^{\mathrm{ab}}$ & $0.983^{a}$ & $2.171^{\text {ab }}$ \\
\hline $1.819^{\mathrm{bc}}$ & $0.713^{b}$ & $2.5^{a b}$ & $1.819^{\mathrm{bc}}$ & $0.732^{b}$ & $2.50^{\mathrm{ab}}$ & $\mathrm{P}_{2}$ \\
\hline $2.406^{a}$ & $0.776^{a b}$ & $3.16^{\mathrm{a}}$ & $2.860^{a}$ & $0.933^{\mathrm{ab}}$ & $3.16^{\mathrm{a}}$ & $F_{1}$ \\
\hline $1.763^{b c}$ & $0.695^{b}$ & $2.66^{\mathrm{ab}}$ & $1.943^{b c}$ & $0.926^{a b}$ & $2.33^{b}$ & $\mathrm{~F}_{2}$ \\
\hline $1.598^{\mathrm{c}}$ & $0.658^{b}$ & $2.50^{\mathrm{ab}}$ & $1.546^{\mathrm{c}}$ & $0.774^{\mathrm{b}}$ & $2.00^{b}$ & $\mathrm{BC}_{1}$ \\
\hline $1.568^{\mathrm{c}}$ & $0.686^{\mathrm{b}}$ & $2.33^{b}$ & $1.541^{\mathrm{c}}$ & $0.672^{b}$ & $2.33^{b}$ & $\mathrm{BC}_{2}$ \\
\hline $2.008^{a b c}$ & $0.871^{\mathrm{ab}}$ & $2.33^{b}$ & $2.008^{b c}$ & $0.871^{\mathrm{b}}$ & $2.33^{b}$ & Gallia1 \\
\hline \multicolumn{3}{|c|}{ Cross $10(4 \times 5)$} & \multicolumn{4}{|c|}{ Cross $5(2 \times 3)$} \\
\hline $2.307^{\text {ab }}$ & $0.739^{b}$ & $3.16^{a}$ & $2.150^{\mathrm{ab}}$ & $0.765^{b}$ & $2.83^{a}$ & $P_{1}$ \\
\hline $1.819^{\mathrm{ab}}$ & $0.713^{b}$ & $2.50^{\mathrm{a}}$ & $2.171^{\mathrm{ab}}$ & $0.983^{a b}$ & $2.50^{\mathrm{a}}$ & $\mathrm{P}_{2}$ \\
\hline $2.213^{\mathrm{ab}}$ & $0.925^{a b}$ & $2.50^{a}$ & $1.666^{\mathrm{b}}$ & $0.716^{\mathrm{b}}$ & $2.33^{a}$ & $F_{1}$ \\
\hline $2.438^{a}$ & $1.067^{\mathrm{a}}$ & $2.33^{a}$ & $1.605^{b}$ & $0.790^{b}$ & $2.33^{a}$ & $\mathrm{~F}_{2}$ \\
\hline $2.154^{\mathrm{ab}}$ & $0.879^{a b}$ & $2.66^{a}$ & $2.172^{a b}$ & $1.027^{a b}$ & $2.15^{a}$ & $\mathrm{BC}_{1}$ \\
\hline $1.690^{\mathrm{b}}$ & $0.716^{\mathrm{b}}$ & $2.50^{a}$ & $2.811^{a}$ & $1.250^{\mathrm{a}}$ & $2.33^{a}$ & $\mathrm{BC}_{2}$ \\
\hline $2.008^{a b}$ & $0.871^{a b}$ & $2.33^{a}$ & $2.008^{b}$ & $0.871^{a b}$ & $2.33^{a}$ & Gallia1 \\
\hline
\end{tabular}

${ }^{*}$ Means with different superscripts in a column are significantly different at $(P<0.05)$, using Duncan's Multiple Rang Test. 
Table (5): Mean performances of 5 parents, $10 F_{1}, 10 F_{2}, 10 B_{1} P_{1}$ and 10 $\mathrm{BC}_{1} \mathrm{P}_{2}$ sweet melon crosses and a check cultivar for the studied fruit characteristics (over two years of 2013 and 2014)

\begin{tabular}{|c|c|c|c|c|c|}
\hline $\begin{array}{c}\text { Moisture } \\
\text { content (\%) }\end{array}$ & T.S.S (\%) & $\begin{array}{l}\text { Fruit netting } \\
\text { degree }\end{array}$ & $\begin{array}{l}\text { Fruit shape } \\
\text { index }\end{array}$ & $\begin{array}{c}\text { Fruit flesh } \\
\text { thickness } \\
(\%)\end{array}$ & genotypes \\
\hline \multicolumn{6}{|c|}{ Cross $1(1 \times 2)$} \\
\hline $93.77^{a}$ & $12.16^{\mathrm{c}}$ & $7.66^{b}$ & $1.54^{\mathrm{a}}$ & $58.46^{\mathrm{C}_{*}}$ & $\mathrm{P}_{1}$ \\
\hline $91.70^{\mathrm{ab}}$ & $14.33^{\mathrm{ab}}$ & $9.33^{a b}$ & $1.04^{c}$ & $62.39^{b c}$ & $\mathrm{P}_{2}$ \\
\hline $91.58^{\mathrm{ab}}$ & $14.66^{\mathrm{ab}}$ & $10.00^{\mathrm{a}}$ & $0.99^{\mathrm{cd}}$ & $64.32^{a b}$ & $\mathrm{~F}_{1}$ \\
\hline $91.42^{\mathrm{abc}}$ & $14.06^{\mathrm{ab}}$ & $8.33^{a b}$ & $1.21^{b}$ & $68.58^{a b}$ & $\mathrm{~F}_{2}$ \\
\hline $90.34^{b c}$ & $14.58^{\mathrm{ab}}$ & $9.66^{a}$ & $1.06^{c}$ & $66.58^{a b}$ & $\mathrm{BC}_{1}$ \\
\hline $88.23^{c}$ & $15.26^{a}$ & $8.16^{a b}$ & $1.06^{c}$ & $67.37^{a b}$ & $\mathrm{BC}_{2}$ \\
\hline $90.33^{b c}$ & $13.66^{\mathrm{b}}$ & $9.66^{\mathrm{a}}$ & $0.90^{d}$ & $69.20^{\mathrm{a}}$ & Gallia1 \\
\hline \multicolumn{6}{|c|}{ Cross $2(1 \times 3)$} \\
\hline $93.77^{\mathrm{a}}$ & $12.16^{c}$ & $7.66^{b}$ & $1.54^{a}$ & $58.46^{\mathrm{C}}$ & $P_{1}$ \\
\hline $92.72^{\mathrm{ab}}$ & $14.58^{a b}$ & $9.50^{\mathrm{a}}$ & $1.11^{b}$ & $63.64^{b}$ & $\mathrm{P}_{2}$ \\
\hline 91.22 ab & $15.03^{a}$ & $10.00^{\mathrm{a}}$ & $1.27^{b}$ & $64.53^{a b}$ & $\mathrm{~F}_{1}$ \\
\hline $91.81^{\mathrm{ab}}$ & $14.6^{\mathrm{ab}}$ & $9.33^{a}$ & $1.24^{b}$ & $64.74^{\mathrm{ab}}$ & $\mathrm{F}_{2}$ \\
\hline $91.36^{\mathrm{ab}}$ & $13.08^{b c}$ & $9.16^{\mathrm{a}}$ & $1.18^{b}$ & $67.77^{\mathrm{ab}}$ & $\mathrm{BC}_{1}$ \\
\hline $91.05^{b}$ & $13.83^{a b}$ & $8.66^{a b}$ & $1.13^{b}$ & $65.67^{a b}$ & $\mathrm{BC}_{2}$ \\
\hline $90.33^{b}$ & $13.66^{\mathrm{ab}}$ & $10.00^{\mathrm{a}}$ & $0.90^{\mathrm{c}}$ & $69.20^{\mathrm{a}}$ & Gallia1 \\
\hline \multicolumn{6}{|c|}{ Cross $3(1 \times 4)$} \\
\hline $93.77^{a}$ & $12.16^{d}$ & $7.66^{b}$ & $1.54^{\mathrm{a}}$ & $58.63^{c}$ & $\mathrm{P}_{1}$ \\
\hline $90.50^{\mathrm{bcd}}$ & $14.01^{b c}$ & $10.00^{\mathrm{a}}$ & $0.97^{c}$ & $63.28^{\mathrm{b}}$ & $\mathrm{P}_{2}$ \\
\hline $91.06^{\mathrm{bc}}$ & $12.83^{c d}$ & $10.00^{\mathrm{a}}$ & $1.06^{\mathrm{b}}$ & $66.65^{a b}$ & $\mathrm{~F}_{1}$ \\
\hline $88.17^{d}$ & $16.13^{a}$ & $9.83^{a}$ & $1.01^{b c}$ & $66.03^{a b}$ & $\mathrm{~F}_{2}$ \\
\hline $88.89^{c d}$ & $14.61^{\mathrm{ab}}$ & $10.00^{\mathrm{a}}$ & $0.95^{c}$ & $66.50^{\mathrm{ab}}$ & $\mathrm{BC}_{1}$ \\
\hline $91.52^{b}$ & $15.96^{a}$ & $9.83^{a}$ & $0.94^{\mathrm{c}}$ & $66.81^{a b}$ & $\mathrm{BC}_{2}$ \\
\hline $90.33^{\mathrm{bcd}}$ & $13.66^{\mathrm{bcd}}$ & $10.00^{\mathrm{a}}$ & $0.90^{\mathrm{c}}$ & $69.20^{\mathrm{a}}$ & Gallia1 \\
\hline \multicolumn{6}{|c|}{ Cross $4(1 \times 5)$} \\
\hline $93.77^{a}$ & $12.16^{d}$ & $7.66^{c}$ & $1.54^{a}$ & $58.63^{b}$ & $\mathrm{P}_{1}$ \\
\hline $89.90^{c}$ & $14.66^{\mathrm{bc}}$ & $9.33^{\mathrm{ab}}$ & $0.93^{\mathrm{cd}}$ & $64.34^{\mathrm{ab}}$ & $\mathrm{P}_{2}$ \\
\hline $91.36^{\mathrm{bc}}$ & $16.86^{a}$ & $9.83^{a}$ & $1.09^{b}$ & $67.65^{\mathrm{a}}$ & $\mathrm{F}_{1}$ \\
\hline $89.50^{c}$ & $16.91^{\mathrm{a}}$ & $9.83^{a}$ & $1.05^{\mathrm{bc}}$ & $65.72^{a}$ & $\mathrm{~F}_{2}$ \\
\hline $92.32^{\mathrm{ab}}$ & $13.43^{c d}$ & $7.66^{c}$ & $1.00^{\mathrm{bcd}}$ & $68.98^{a b}$ & $\mathrm{BC}_{1}$ \\
\hline $90.52^{b c}$ & $15.55^{\mathrm{ab}}$ & $8.16^{b c}$ & $1.01^{\mathrm{bcd}}$ & $63.96^{\mathrm{ab}}$ & $\mathrm{BC}_{2}$ \\
\hline $90.33^{b c}$ & $13.66^{c d}$ & $10.00^{\mathrm{a}}$ & $0.90^{d}$ & $69.20^{\mathrm{a}}$ & Gallia1 \\
\hline \multicolumn{6}{|c|}{ Cross $5(2 \times 3)$} \\
\hline $91.71^{\mathrm{ab}}$ & $14.33^{b c}$ & $9.33^{a}$ & $1.04^{b c}$ & $62.39^{c}$ & $\mathrm{P}_{1}$ \\
\hline $92.73^{a}$ & $14.58^{\mathrm{abc}}$ & $9.50^{\mathrm{a}}$ & $1.11^{\mathrm{b}}$ & $63.64^{b c}$ & $\mathrm{P}_{2}$ \\
\hline $89.54^{b}$ & $15.09^{a b}$ & $9.33^{a}$ & $0.93^{c}$ & $65.14^{a b c}$ & $F_{1}$ \\
\hline $89.91^{\mathrm{ab}}$ & $16.33^{a}$ & $10.00^{a}$ & $1.01^{b c}$ & $65.98^{a b c}$ & $\mathrm{~F}_{2}$ \\
\hline $91.93^{\mathrm{ab}}$ & $13.13^{c}$ & $9.66^{a}$ & $0.96^{c}$ & $64.45^{b c}$ & $\mathrm{BC}_{1}$ \\
\hline $90.72^{a b}$ & $12.83^{c}$ & $9.66^{a}$ & $1.25^{\mathrm{a}}$ & $68.19^{a b}$ & $\mathrm{BC}_{2}$ \\
\hline $91.84^{\mathrm{ab}}$ & $13.67^{\mathrm{bc}}$ & $10.00^{\mathrm{a}}$ & $0.90^{\mathrm{C}}$ & $69.20^{\mathrm{a}}$ & Gallia1 \\
\hline
\end{tabular}

* Means with different superscripts in a column are significantly different at $(P<0.05)$, using Duncan's Multiple Rang Test.

To be Conted... 
Table (5) Cont'

\begin{tabular}{|c|c|c|c|c|c|}
\hline $\begin{array}{c}\text { Moisture } \\
\text { content \% }\end{array}$ & T.S.S \% & $\begin{array}{c}\text { Fruit } \\
\text { netting } \\
\text { degree }\end{array}$ & $\begin{array}{c}\text { Fruit shape } \\
\text { index }\end{array}$ & $\begin{array}{c}\text { Fruit flesh } \\
\text { thickness } \\
\%\end{array}$ & genotypes \\
\hline \multicolumn{6}{|c|}{ Cross $6(2 \times 4)$} \\
\hline $91.71^{a b}$ & $14.33^{\mathrm{ab}}$ & $9.33^{a b}$ & $1.04^{a b}$ & $62.39^{\mathrm{bc} *}$ & $P_{1}$ \\
\hline $90.51^{\mathrm{ab}}$ & $14.02^{\mathrm{abc}}$ & $10.00^{a}$ & $0.97^{a b c}$ & $63.28^{b c}$ & $\mathrm{P}_{2}$ \\
\hline $91.50^{\mathrm{ab}}$ & $14.97^{\mathrm{ab}}$ & $10.00^{a}$ & $1.08^{a}$ & $67.28^{a b}$ & $\mathrm{~F}_{1}$ \\
\hline $89.15^{b}$ & $15.28^{a}$ & $10.00^{a}$ & $1.03^{a b c}$ & $67.03^{a b}$ & $\mathrm{~F}_{2}$ \\
\hline $91.17^{\mathrm{ab}}$ & $15.32^{a}$ & $9.16^{b}$ & $1.03^{a b c}$ & $62.62^{\mathrm{bc}}$ & $\mathrm{BC}_{1}$ \\
\hline $92.36^{\mathrm{a}}$ & $12.77^{c}$ & $7.16^{c}$ & $0.89^{c}$ & $61.36^{c}$ & $\mathrm{BC}_{2}$ \\
\hline $91.84^{\mathrm{ab}}$ & $13.67^{\mathrm{bc}}$ & $10.00^{a}$ & $0.90^{\mathrm{bc}}$ & $69.20^{\mathrm{a}}$ & Gallia1 \\
\hline \multicolumn{6}{|c|}{ Cross $7(2 \times 5)$} \\
\hline $91.71^{a}$ & $14.33^{a}$ & $9.33^{a}$ & $1.04^{\mathrm{a}}$ & $62.39^{b c}$ & $\mathrm{P}_{1}$ \\
\hline $89.90^{a}$ & $14.67^{\mathrm{a}}$ & $9.33^{a}$ & $0.93^{c}$ & $66.84^{\mathrm{abc}}$ & $\mathrm{P}_{2}$ \\
\hline $90.71^{a}$ & $13.53^{a}$ & $9.67^{a}$ & $0.94^{b c}$ & $68.17^{\mathrm{ab}}$ & $\mathrm{F}_{1}$ \\
\hline $91.66^{a}$ & $13.50^{a}$ & $9.83^{a}$ & $1.03^{a b}$ & $68.20^{a b}$ & $\mathrm{~F}_{2}$ \\
\hline $89.90^{a}$ & $13.80^{a}$ & $9.67^{a}$ & $0.92^{c}$ & $60.94^{c}$ & $\mathrm{BC}_{1}$ \\
\hline $91.90^{\mathrm{a}}$ & $12.82^{a}$ & $9.50^{a}$ & $1.09^{a}$ & $66.22^{a b c}$ & $\mathrm{BC}_{2}$ \\
\hline $91.84^{a}$ & $13.67^{a}$ & $10.00^{a}$ & $0.90^{c}$ & $69.20^{\mathrm{a}}$ & Gallia1 \\
\hline \multicolumn{6}{|c|}{ Cross $8(3 \times 4)$} \\
\hline $83.72^{b}$ & $14.58^{a b}$ & $9.50^{a b}$ & $1.11^{\mathrm{a}}$ & $63.64^{b c}$ & $P_{1}$ \\
\hline $90.41^{a}$ & $14.01^{\mathrm{b}}$ & $10.00^{a}$ & $0.96^{\mathrm{bc}}$ & $63.28^{b c}$ & $\mathrm{P}_{2}$ \\
\hline $90.51^{\mathrm{a}}$ & $15.71^{\mathrm{a}}$ & $10.00^{a}$ & $1.06^{a b}$ & $63.02^{b c}$ & $\mathrm{~F}_{1}$ \\
\hline $90.53^{a}$ & $13.91^{\mathrm{b}}$ & $10.00^{a}$ & $0.96^{\mathrm{bc}}$ & $66.43^{\mathrm{ab}}$ & $\mathrm{F}_{2}$ \\
\hline $90.50^{\mathrm{a}}$ & $15.66^{a}$ & $8.66^{b}$ & $0.95^{\mathrm{bc}}$ & $63.22^{b c}$ & $\mathrm{BC}_{1}$ \\
\hline $90.67^{a}$ & $15.68^{a}$ & $9.50^{\mathrm{ab}}$ & $0.94^{c}$ & $60.68^{c}$ & $\mathrm{BC}_{2}$ \\
\hline $90.33^{a}$ & $13.66^{b}$ & $10.00^{a}$ & $0.90^{c}$ & $68.20^{a}$ & Gallia1 \\
\hline \multicolumn{6}{|c|}{ Cross $9(3 \times 5)$} \\
\hline $92.72^{a}$ & $14.58^{\mathrm{a}}$ & $9.5^{\mathrm{ab}}$ & $1.11^{\mathrm{a}}$ & $63.64^{b c}$ & $\mathrm{P}_{1}$ \\
\hline $89.90^{b}$ & $14.66^{\mathrm{a}}$ & $9.33^{a b}$ & $0.93^{a}$ & $65.34^{\mathrm{abc}}$ & $\mathrm{P}_{2}$ \\
\hline $91.72^{a b}$ & $14.41^{\mathrm{a}}$ & $10.00^{a}$ & $1.00^{a}$ & $70.00^{a}$ & $\mathrm{~F}_{1}$ \\
\hline $90.15^{a b}$ & $15.11^{\mathrm{a}}$ & $9.00^{\mathrm{b}}$ & $0.97^{\mathrm{a}}$ & $62.47^{c}$ & $\mathrm{~F}_{2}$ \\
\hline $90.81^{a b}$ & $15.13^{\mathrm{a}}$ & $9.83^{a}$ & $0.99^{a}$ & $65.22^{a b c}$ & $\mathrm{BC}_{1}$ \\
\hline $89.19^{b}$ & $14.38^{a}$ & $10.00^{\mathrm{a}}$ & $0.98^{a}$ & $66.11^{\mathrm{abc}}$ & $\mathrm{BC}_{2}$ \\
\hline $90.33^{a b}$ & $13.66^{a}$ & $10.00^{a}$ & $0.90^{\mathrm{a}}$ & $68.20^{a b}$ & Gallia1 \\
\hline \multicolumn{6}{|c|}{ Cross $10(4 \times 5)$} \\
\hline $90.50^{a b}$ & $14.01^{\text {ab }}$ & $10.00^{a}$ & $0.97^{\mathrm{b}}$ & $63.28^{a b}$ & $\mathrm{P}_{1}$ \\
\hline $89.90^{b}$ & $14.66^{a b}$ & $9.33^{b}$ & $0.93^{b}$ & $65.34^{a b}$ & $\mathrm{P}_{2}$ \\
\hline $92.27^{\mathrm{a}}$ & $15.50^{\mathrm{a}}$ & $9.83^{a}$ & $0.93^{b}$ & $61.97^{b}$ & $\mathrm{~F}_{1}$ \\
\hline $91.49^{a b}$ & $14.60^{a b}$ & $9.83^{a}$ & $1.15^{\mathrm{a}}$ & $62.22^{b}$ & $\mathrm{~F}_{2}$ \\
\hline $92.32^{a}$ & $14.26^{a b}$ & $9.66^{a}$ & $1.09^{a}$ & $63.95^{\mathrm{ab}}$ & $\mathrm{BC}_{1}$ \\
\hline $90.10^{b}$ & $15.16^{\mathrm{ab}}$ & $8.66^{b}$ & $0.97^{\mathrm{b}}$ & $61.93^{b}$ & $\mathrm{BC}_{2}$ \\
\hline $90.33^{\mathrm{ab}}$ & $13.66^{\mathrm{b}}$ & $10.00^{a}$ & $0.90^{\mathrm{b}}$ & $68.87^{a}$ & Gallia1 \\
\hline
\end{tabular}

* Means with different superscripts in a column are significantly different at $(P<0.05)$, using Duncan's Multiple Rang Test. 


\section{Genetical parameters}

\section{Type of gene action}

The data of the genetic analysis which give estimation on population mean values (m), additive gene action (a), dominance gene action (d) and the three epistatic effect ; i.e. additive $x$ additive (aa), additive $x$ dominance (dd) and dominance $x$ dominance for the tested characters are presented in Tables $(6,7$ and 8).

The data of plant height showed significant variation for all the crosses; therefore, to improve this trait, selection in the advanced selfed generation on the basis of family mean performance would be effective. With this respect, the crosses $1 \times 3,1 \times 4,3 \times 4$ and $3 \times 5$ gave, highly, significant values for the additive gene effects indicating that, selection for the longest plant would be effective in these crosses. The dominance effects were found to be highly significant with positive values for all the hybrids except of the hybrid $4 \times 5$, these result indicated that, the dominance gene effect was important in the inheritance of this characters. The additive $x$ additive interaction found to be highly significant with positive values for all the crosses except of importance of the crosses $3 \times 5$ and $4 \times 5$ indicating the importance of additive $x$ additive gene action in the inheritance of this trait. The additive $x$ dominance interaction was found to be highly significant for all the crosses with positive values for the crosses $1 \times 3$, $1 \times 4,3 \times 4$ and $3 \times 5$ and negative value for the crosses $1 \times 2,1 \times 5,2 \times 3,2 \times 4,2 \times 5$ and $4 \times 5$. This results indicating that, the selection for this trait in the early generation would be not effective. The dominance $x$ dominance was found to be highly significant for all the crosses with positive values for the crosses $1 \times 3$, $2 \times 4,3 \times 4,3 \times 5$ and $4 \times 5$ and negative values for crosses $1 \times 2,1 \times 4,1 \times 5,2 \times 3$ and $2 \times 5$, this results indicating that the dominance gene For the number of branches per plant character, the additive gene effect exhibited insignificant values for all the crosses. The results showed that, the dominance $x$ dominance epistatic were recorded significant and highly significant values for all the crosses.

The data presented in Table (6) for the no. of branches per plant trait revealed that the additive gene effect exhibited insignificant values for all the crosses. The results showed that, the mean value of the fruit maturity date character recorded highly significant and significant only for the two hybrids $1 \times 5$ and $2 \times 3$, respectively, indicating that, the population mean values might be effective as indicator for selection for this trait. while the dominance gene effect was significant with positive values for the crosses $1 \times 2,1 \times 4,1 \times 5,2 \times 4,2 \times 5,3 \times 4$, $3 \times 5$ and $4 \times 5$ indicating that the dominance gene effect had the main role in the inheritance of this character. The additive $x$ additive (aa) epistatic found to be significant and highly significant with positive values for the crosses $1 \times 2,1 \times 4$, $2 \times 4,2 \times 5$ and $4 \times 5$. The dominance $x$ dominance epistatic was found to be significant an highly significant with positive values for the crosses $1 \times 3$ and $2 \times 3$ , while the crosses $1 \times 2,1 \times 4,1 \times 5,2 \times 4,2 \times 5,3 \times 4,3 \times 5$ and $4 \times 5$ exhibited highly significant with negative value. These results showed that the dominance effect have the main role in the inheritance of this character. Dominance $x$ dominance epistatic were recorded significant and highly significant values for all the crosses. 
Table (6): Gene action of 5 parents, $10 F_{1}, 10 F_{2}, 10 B C_{1} P_{1}$ and $10 B C_{1} P_{2}$ sweet melon crosses for the studied vegetative characters, flowering date and maturity date

\begin{tabular}{|c|c|c|c|c|c|c|c|c|}
\hline $\begin{array}{l}\text { Maturity } \\
\text { date } \\
\text { (day) }\end{array}$ & $\begin{array}{c}\text { Flowering } \\
\text { date } \\
\text { (day) }\end{array}$ & $\begin{array}{c}\text { No. of } \\
\text { branches } \\
\text { per plant }\end{array}$ & $\begin{array}{l}\text { Plant } \\
\text { height } \\
(\mathrm{cm})\end{array}$ & $\begin{array}{l}\text { Maturity } \\
\text { date } \\
\text { (day) }\end{array}$ & $\begin{array}{l}\text { Flowering } \\
\text { date } \\
\text { (day) }\end{array}$ & $\begin{array}{c}\text { No. of } \\
\text { branches } \\
\text { per plant }\end{array}$ & $\begin{array}{c}\text { Plant } \\
\text { height } \\
(\mathrm{cm})\end{array}$ & $\begin{array}{l}\text { Gene } \\
\text { action }\end{array}$ \\
\hline \multicolumn{4}{|c|}{ Cross $6(2 x 4)$} & \multicolumn{5}{|c|}{ Cross $1(1 \times 2)$} \\
\hline 80.33 & 39.83 & 4.50 & $198.5^{\star \star}$ & 73.33 & 41.00 & 3.83 & $199.3^{\star \star}$ & $m$ \\
\hline-15.33 & -2.27 & 0.17 & $-10.17^{* *}$ & 4.83 & -0.5 & -0.33 & $-14.33^{* *}$ & $a$ \\
\hline $14.83^{\star *}$ & $8.20^{* *}$ & $-2.42^{*}$ & $59.08^{\star \star}$ & $20.58^{\star *}$ & -3.65 & 2.44 & $111.25^{\star *}$ & $d$ \\
\hline $17.33^{\star \star}$ & $5.86^{* *}$ & $-2.33^{*}$ & $18.33^{* *}$ & $29.33^{\star *}$ & $-1.00^{\star *}$ & 2.02 & $82.67^{\star \star}$ & $a a$ \\
\hline-14.83 & $-1.43^{\star}$ & 0.42 & $-11.25^{\star *}$ & $6.92^{\star}$ & -3.85 & -0.75 & $-13.58^{* *}$ & $a d$ \\
\hline$-9.67^{* *}$ & $-21.07^{* *}$ & $2.83^{\star *}$ & $154.50^{\star *}$ & $-71.50^{* *}$ & $-15.37^{\star *}$ & $-1.50^{*}$ & $-0.50^{\star *}$ & $d d$ \\
\hline \multicolumn{4}{|c|}{ Cross 7 (2x 5) } & \multicolumn{5}{|c|}{ Cross $2(1 \times 3)$} \\
\hline 80.33 & 38.0 & 3.67 & $190.83^{\star *}$ & 81.17 & 38.83 & 3.83 & $217.67^{* *}$ & $m$ \\
\hline 15.17 & 5.00 & 0.50 & $-23.33^{\star *}$ & 5.17 & 2.67 & -0.50 & $8.33^{\star *}$ & $a$ \\
\hline $15.75^{\star \star}$ & $28.0^{* *}$ & -0.76 & $160.58^{* *}$ & $-34.67^{* *}$ & $-5.83^{\star \star}$ & 3.17 & $71.70^{\star *}$ & $d$ \\
\hline $15.66^{* *}$ & $24^{\star *}$ & -1.00 & $134.66^{* *}$ & $-27.67^{* *}$ & $0.67^{* *}$ & 3.00 & $38.67^{* *}$ & $a a$ \\
\hline 15.58 & 5.50 & 0.33 & $-27.92^{\star *}$ & 3.00 & 0.67 & -0.67 & $7.47^{* *}$ & $a d$ \\
\hline$-31.50^{* \star}$ & $-48.88^{\star \star}$ & $2.67^{\star}$ & $-91.17^{* *}$ & $25.33^{\star \star}$ & $-7.00^{* *}$ & -3.00 & $16.60^{\star *}$ & $d d$ \\
\hline \multicolumn{4}{|c|}{ Cross $8(3 \times 4)$} & \multicolumn{5}{|c|}{ Cross $3(1 \times 4)$} \\
\hline 90.83 & 40.67 & 4.17 & $211.67^{* *}$ & 76.50 & 37.00 & 3.00 & $212.33^{\star *}$ & $m$ \\
\hline-4.67 & -2.50 & 0.17 & $1.67^{* *}$ & -6.67 & 2.83 & -0.33 & $14.50^{* *}$ & $a$ \\
\hline $6.75^{\star *}$ & $9.33^{* *}$ & -1.50 & $90.67^{* *}$ & $15.50^{*}$ & $13.58^{*}$ & 6.17 & $81.67^{\star *}$ & $d$ \\
\hline$-2.00^{* *}$ & $7.66^{* *}$ & -1.66 & $96.66^{\star \star}$ & $16.67^{*}$ & $17.67^{*}$ & 6.67 & $58.33^{* *}$ & aa \\
\hline$-3.08^{*}$ & -3.17 & 0.17 & $2.17^{* *}$ & -6.50 & 0.42 & -0.50 & $14.17^{\star *}$ & ad \\
\hline$-8.83^{\star *}$ & $23.33^{\star *}$ & $4.33^{*}$ & $45.33^{* *}$ & $-23.67^{*}$ & $-31.83^{\star *}$ & $-8.33^{\star}$ & $-18.67^{* *}$ & $d d$ \\
\hline \multicolumn{4}{|c|}{ Cross $9(3 \times 5)$} & \multicolumn{5}{|c|}{ Cross $4(1 \times 5)$} \\
\hline 84.33 & 40.67 & 3.67 & $221.67^{* *}$ & $88.33^{* \star}$ & 38.83 & 4.33 & $207.83^{* *}$ & $m$ \\
\hline-6.33 & -8.33 & 0.17 & $7.67^{\star \star}$ & -0.50 & 1.33 & 0.50 & $-33.0^{\star *}$ & $a$ \\
\hline $10.17^{*}$ & $12.58^{*}$ & 3.08 & $12.17^{* \star}$ & $6.17^{* *}$ & $-0.17^{* *}$ & -0.08 & $130.17^{* *}$ & $d$ \\
\hline 14.00 & $16.00^{*}$ & 2.33 & $-20.66^{* *}$ & $11.67^{\star *}$ & $4.00^{\star \star}$ & -0.33 & $89.33^{\star *}$ & $a a$ \\
\hline-4.17 & -8.42 & -0.25 & $4.67^{* *}$ & 0.50 & -1.50 & -0.08 & $-36.83^{* *}$ & $a d$ \\
\hline$-57.67^{\star}$ & $-50.50^{\star *}$ & $-2.017^{*}$ & $103.67^{\star *}$ & $-69.0^{* *}$ & $-9.67^{\star *}$ & $0.17^{\star}$ & $-41.67^{* *}$ & $d d$ \\
\hline \multicolumn{4}{|c|}{ Cross $10(4 \times 5)$} & \multicolumn{5}{|c|}{ Cross $5(2 \times 3)$} \\
\hline 80.33 & 38.50 & 3.50 & $220.17^{\star *}$ & $94.50^{*}$ & 41.00 & 4.17 & $221.17^{* *}$ & $m$ \\
\hline 4.50 & 1.17 & 0.17 & $-11.67^{\star *}$ & 0.17 & 0.17 & 0.67 & $-22.13^{\star *}$ & $a$ \\
\hline $3.25^{* *}$ & 12.75 & 2.55 & $-1.33^{* *}$ & $-58.42^{* *}$ & $-1.67^{\star *}$ & 0.08 & $73.58^{\star *}$ & $d$ \\
\hline $7.66^{* *}$ & 11.00 & 2.33 & $26.00^{* *}$ & $-54.33^{\star *}$ & $0.33^{* *}$ & 0.00 & $70.00^{\star *}$ & aa \\
\hline 5.08 & 1.25 & -0.25 & $-15.17^{\star \star}$ & -1.25 & 1.67 & 0.92 & $-23.92^{\star *}$ & $a d$ \\
\hline$-23.83^{\star *}$ & -22.17 & $-2.57^{*}$ & $106.00^{* *}$ & $30.83^{* *}$ & $-18.00^{\star *}$ & -0.17 & $-115.8^{\star *}$ & $d d$ \\
\hline
\end{tabular}

${ }^{*},{ }^{* *}$ Significant and highly significant at the 0.05 and 0.01 level of probability, respectively.

( $m, a, d, a a, a d$ and $d d=$ population mean, additive, dominant, additive $\mathrm{x}$ additive, additive $\mathrm{x}$ dominant and dominant $x$ dominant gene action, respectively).

The data of the flowering date character appeared that the additive gene effect exhibited insignificant for all the crosses indicating that the role of the additive gene effect was negligible in the inheritance of this character. The dominance gene effect exhibited significant and highly significant positive value 
for the crosses $1 \times 4,2 \times 4,2 \times 5,3 \times 4$ and $3 \times 5$. The additive $\times$ additive epistatic gene effects ( $\mathrm{aa}$ ) were found to be significant with the positive value for all the crosses, indicating that the additive $x$ additive interaction was important in the inheritance of this trait.

The type of gene action for fruit yield per plant and yield components (No. of fruits per plant and average fruit weight) are presented in Table (7). The mean values of these characters were found to be insignificant for all the tested crosses indicating that the population mean may be not effective as an indicator for selection for these traits. The tabulated data of the No. of fruits per plant showed that the dominance effect exhibited significant values only for the crosses $1 \times 3,2 \times 3$ and $2 \times 4$. The dominance $x$ dominance interaction was found to be significant for all the crosses except for the crosses $1 \times 2$ and $2 \times 5$. These results indicating that the dominance $x$ dominance interaction was more important in the inheritance of this character.

The data of average fruit weight per plant character are presented in Table (8). the additive and dominance effects showed insignificant values for all crosses indicating that the additive and dominance effects might be not effective in the inheritance of this characters, the additive $x$ additive interaction showed insignificant values for all the crosses except for the cross $2 \times 4$ which gave significant with negative value. The additive $x$ dominance interaction was found to be insignificant for all the crosses except of the cross $1 \times 4$ which gave significant negative value.

For total fruit yield per plant characters, the recorded data showed that the additive and dominance effects were found to be insignificant for all the crosses except for the cross $2 \times 5$ which gave significant value for the dominance effect. The additive $x$ additive interaction was found to be insignificant for all the crosses except for the $2 \times 5$ and $3 \times 4$, the dominance $x$ dominance interaction was found to be significant for the crosses $1 \times 4,2 \times 3,2 \times 4$ and $3 \times 4$ indicating the importance of the dominance epistatic in the inheritance of this character for the tested crosses.

\section{Fruit quality characteristics}

Data presented in Table (8) declare these characters of gene action for fruit fresh thickness, the mean values was found to be significant and highly significant for the crosses $1 \times 3,1 \times 4,1 \times 5,2 \times 5$ and $4 \times 5$ indicating that improvement of such a character might be effective depending upon the population mean in the breeding selection. The additive gene action was significant and highly significant for all the tested crosses except for the hybrids $1 \times 3,1 \times 4,2 \times 3$ and $3 \times 5$. Concerning the dominant gene effect; results showed high significant for the tested crosses with highly magnitude values, which may be emphasize that the dominant gene effect was important in the inheritance of this trait. The additive $x$ additive interaction 
Table (7):Gene action of 5 parents, $10 F_{1}, 10 F_{2}, 10 B C_{1} P_{1}$, and $10 B C_{1} P_{2}$ sweet melon crosses for the studied yield and yield components characters

\begin{tabular}{|c|c|c|c|c|c|c|}
\hline $\begin{array}{l}\text { Total fruit yield per plant } \\
\qquad(\mathrm{kg})\end{array}$ & $\begin{array}{l}\text { Average fruit } \\
\text { weight }(\mathrm{kg})\end{array}$ & $\begin{array}{c}\text { Fruits No. } \\
\text { per plant }\end{array}$ & $\begin{array}{c}\text { Total fruit } \\
\text { yield per plant } \\
(\mathrm{kg})\end{array}$ & $\begin{array}{l}\text { Average fruit } \\
\text { weight }(\mathrm{kg})\end{array}$ & $\begin{array}{l}\text { Fruits No. } \\
\text { per plant }\end{array}$ & $\begin{array}{l}\text { Gene } \\
\text { action }\end{array}$ \\
\hline \multicolumn{3}{|c|}{ Cross $6(2 x 4)$} & \multicolumn{4}{|c|}{ Cross $1(1 \times 2)$} \\
\hline 1.830 & 1.100 & 2.00 & 1.610 & 0.758 & 2.17 & $m$ \\
\hline 0.73 & -0.04 & 0.76 & -0.568 & -0.40 & 0.50 & $a$ \\
\hline-0.43 & -1.81 & $1.00^{*}$ & 2.027 & 0.229 & 2.33 & $d$ \\
\hline-1.18 & $-1.91^{*}$ & $0.66^{*}$ & 1.704 & 0.369 & 1.67 & $a a$ \\
\hline 0.81 & -0.22 & 0.83 & -0.568 & -0.612 & 1.00 & $a d$ \\
\hline $5.51^{* *}$ & 3.28 & $3.33^{*}$ & 0.602 & -0.161 & -1.33 & $d d$ \\
\hline \multicolumn{3}{|c|}{ Cross $7(2 \times 5)$} & \multicolumn{4}{|c|}{ Cross $2(1 \times 3)$} \\
\hline 2.280 & 1.020 & 2.33 & 1.780 & 0.900 & 2.17 & $m$ \\
\hline 0.47 & -0.21 & 0.00 & 0.28 & -0.02 & 0.67 & $a$ \\
\hline $0.70^{*}$ & -1.09 & 0.83 & 3.23 & 0.81 & $2.00^{*}$ & $d$ \\
\hline$-1.73^{*}$ & -1.06 & 0.66 & 3.27 & 0.80 & 2.00 & $a a$ \\
\hline-0.63 & -0.23 & -0.17 & 0.38 & -0.12 & 1.00 & $a d$ \\
\hline 2.36 & 0.99 & 3.30 & -4.73 & -0.81 & $-4.00^{*}$ & $d d$ \\
\hline \multicolumn{3}{|c|}{ Cross $8(3 \times 4)$} & \multicolumn{4}{|c|}{ Cross $3(1 \times 4)$} \\
\hline 1.590 & 0.680 & 2.33 & 1.630 & 0.790 & 2.33 & $m$ \\
\hline 0.92 & -0.17 & 0.33 & 0.89 & 0.81 & -0.50 & $a$ \\
\hline 3.02 & 0.40 & -0.17 & 4.29 & 1.61 & 0.50 & $d$ \\
\hline $2.80^{*}$ & 0.45 & 0.00 & 3.90 & 1.63 & 0.33 & aa \\
\hline 0.89 & -0.29 & 0.67 & 0.97 & 0.59 & 0.17 & $a d$ \\
\hline$-2.19^{* *}$ & -0.29 & $1.67^{\star}$ & $-4.61^{*}$ & $-2.63^{*}$ & $0.33^{*}$ & $d d$ \\
\hline \multicolumn{3}{|c|}{ Cross $9(3 \times 5)$} & \multicolumn{4}{|c|}{ Cross $4(1 \times 5)$} \\
\hline 1.760 & 0.700 & 2.67 & 1.890 & 0.93 & 2.17 & $m$ \\
\hline 0.03 & -0.03 & 0.17 & 0.01 & 0.10 & -0.33 & $a$ \\
\hline-0.39 & -0.16 & -0.33 & -0.38 & -0.85 & 1.00 & $d$ \\
\hline 0.72 & -0.09 & -1.00 & -1.25 & -0.84 & 0.00 & $a a$ \\
\hline-0.23 & -0.16 & 0.17 & -0.25 & -0.13 & 0.00 & $a d$ \\
\hline 3.42 & 0.66 & $2.67^{*}$ & 4.77 & 1.71 & $2.00^{*}$ & $d d$ \\
\hline \multicolumn{3}{|c|}{ Cross $10(4 \times 5)$} & \multicolumn{4}{|c|}{ Cross $5(2 \times 3)$} \\
\hline 2.440 & 1.070 & 2.33 & 1.600 & 0.790 & 2.33 & $m$ \\
\hline 0.46 & 0.16 & 0.17 & -0.69 & -0.22 & -0.17 & $a$ \\
\hline-1.58 & -0.88 & 0.33 & 2.96 & 1.24 & $-0.67^{*}$ & $d$ \\
\hline-2.06 & -1.08 & 0.33 & 3.55 & 1.39 & -0.33 & $a a$ \\
\hline 0.22 & 0.15 & -0.17 & -0.54 & -0.11 & -0.33 & $a d$ \\
\hline 3.60 & 1.19 & $1.33^{\star \star}$ & $-5.70^{\star}$ & -2.77 & $1.33^{*}$ & $d d$ \\
\hline
\end{tabular}

*, ** Significant and highly significant at the 0.05 and 0.01 level of probability, respectively.

( $m, a, d, a a, a d$ and $d d=$ population mean, additive, dominant, additive $\mathrm{x}$ additive, additive $\mathrm{x}$ dominant and dominant $x$ dominant gene action, respectively). 
showed highly positive and negative magnitude values with highly significant values in most of the tested crosses except for the cross $2 \times 3$, indicating that the duplicate additive epistatic was more important in the inheritance of this character. Concerning the interaction additive $x$ dominant the results showed significant and highly significant values for all the crosses except for the crosses $2 \times 3$ and $3 \times 5$. The non-allelic interaction dominant $x$ dominant was found to be significant and highly significant with positive values for the crosses $1 \times 5,2 \times 4$, $2 \times 5$ and $3 \times 4$.

For fruit shape index trait, all the evaluated crosses exhibited insignificant values for the six parameters; i.e., mean values, additive gene effect, dominant gene effect, additive $x$ additive, additive $x$ dominant and dominant $x$ dominant. These results suggesting that the improvement of this character in the early generation would be not effective. The data of fruit netting degree showed the additive gene effect was found to be insignificant for all the evaluated crosses, while the dominant gene effect showed highly significant positive value only for the crosses $1 \times 2,1 \times 3$ and $2 \times 3$. The additive $\times$ additive interaction showed significant and highly significant only for the crosses $1 \times 2,1 \times 3$ and $2 \times 3$. Dominance duplicate epistatic was found to be significant and highly significant with negative value for the crosses $1 \times 3$ and $2 \times 3$ while the crosses $1 \times 5,2 \times 4$, $2 \times 5,3 \times 4$ and $4 \times 5$ exhibited positive significant and highly significant values. The non- allelic interaction additive $x$ additive showed significant and highly significant values for all the crosses except for the cross $1 \times 5,2 \times 5$ and $3 \times 5$.

The total soluble solids character showed that the additive gene action exhibited non- significant values for all the evaluated crosses; while the dominance gene action gave significant and highly significant values for most of the tested crosses except for the hybrid $2 \times 4$. These results indicated that the dominance gene effect was more important than the additive gene effect in the inheritance of this character. Concerning the duplicate additive epistatic, the

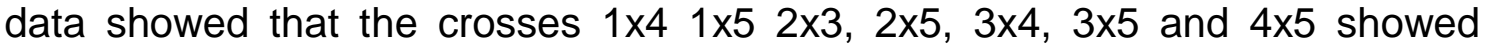
significant and highly significant values. On the other hand, the dominance duplicate interaction was found to be highly significant for most of the tested crosses, except for the hybrid $3 \times 5$.

The mean values of the fruit moisture content trait was found to be insignificant for most of the tested crosses, except for the cross $3 \times 4$ where it gave significant value. The dominant gene action exhibited highly significant with high magnitude values for all the crosses indicating that the dominant gene action was more important than the additive gene effect in the improvement of this character. As for the duplication additive interaction, the results showed that all the crosses exhibited highly significant positive and negative values except for the cross $2 \times 4$ which gave, significantly, positive value. The dominant $x$ dominant interaction showed highly significant values for all the tested crosses. Similar results were reported by Fernaindez et al. (2009) and Reddy et al. (2013`). 
Table (8): Gene action of 5 parents, $10 F_{1}, 10 F_{2}, 10 B C_{1} P_{1}$, and $10 B C_{1} P_{2}$ sweet melon crosses for the studied fruit characteristics

\begin{tabular}{|c|c|c|c|c|c|}
\hline $\begin{array}{c}\text { Moisture } \\
\text { content (\%) }\end{array}$ & T.S.S (\%) & $\begin{array}{c}\text { Fruit } \\
\text { netting } \\
\text { degree }\end{array}$ & $\begin{array}{l}\text { Fruit shape } \\
\text { index }\end{array}$ & $\begin{array}{c}\text { Fruit flesh } \\
\text { thickness } \\
(\%)\end{array}$ & Gene action \\
\hline \multicolumn{6}{|c|}{ Cross $1(1 \times 2)$} \\
\hline 91.43 & 14.07 & 8.33 & 1.22 & 66.67 & $m$ \\
\hline 0.58 & -0.35 & 1.50 & 0.01 & $0.53^{\star *}$ & $a$ \\
\hline$-6.79^{* *}$ & $4.15^{\star}$ & $2.63^{\star *}$ & -0.94 & $7.45^{\star *}$ & $d$ \\
\hline$-5.63^{* *}$ & 2.77 & $2.33^{\star *}$ & -0.64 & $4.27^{\star *}$ & $a a$ \\
\hline-0.45 & 0.77 & $1.79^{*}$ & -0.24 & $1.45^{\star \star}$ & $a d$ \\
\hline $14.23^{\star *}$ & $-5.90^{*}$ & -1.25 & 0.98 & $-26.10^{* *}$ & $d d$ \\
\hline \multicolumn{6}{|c|}{ Cross $2(1 \times 3)$} \\
\hline 91.81 & 14.60 & 8.67 & 1.25 & $65.80^{* *}$ & $m$ \\
\hline 0.32 & -0.74 & 0.50 & 0.05 & 0.30 & $a$ \\
\hline$-4.40^{* *}$ & $-2.92^{*}$ & $1.75^{\star *}$ & -0.41 & $11.58^{* *}$ & $d$ \\
\hline$-2.37^{* *}$ & -4.57 & $1.00^{* *}$ & 0.36 & $5.27^{\star *}$ & aa \\
\hline-0.21 & 0.47 & 1.42 & -0.16 & $2.05^{*}$ & $a d$ \\
\hline $0.45^{\star *}$ & $7.58^{* *}$ & $-0.83^{* *}$ & 0.93 & $-15.17^{*}$ & $d d$ \\
\hline \multicolumn{6}{|c|}{ Cross $3(1 \times 4)$} \\
\hline 88.14 & 14.80 & 10.00 & 1.02 & $66.13^{*}$ & $m$ \\
\hline-2.63 & -1.35 & 0.17 & 0.01 & 1.00 & $a$ \\
\hline $7.17^{\star *}$ & $3.14^{\star *}$ & 0.67 & -0.45 & $9.48^{\star *}$ & $d$ \\
\hline $8.28^{* *}$ & $1.97^{\star *}$ & -0.33 & -0.25 & $3.33^{\star *}$ & aa \\
\hline-4.26 & -0.42 & 1.33 & -0.28 & $2.15^{\star}$ & $a d$ \\
\hline$-2.76^{* *}$ & $-8.42^{* *}$ & -2.00 & 1.08 & $-15.37^{* *}$ & $d d$ \\
\hline \multicolumn{6}{|c|}{ Cross $4(1 \times 5)$} \\
\hline 89.50 & 15.72 & 9.83 & 1.06 & $66.00^{*}$ & $m$ \\
\hline 1.69 & -1.28 & -0.50 & -0.01 & $2.37^{\star *}$ & $a$ \\
\hline $7.47^{* *}$ & $0.22^{* *}$ & -6.33 & -0.33 & $-1.57^{* *}$ & $d$ \\
\hline $7.93^{\star *}$ & $-3.23^{*}$ & -7.67 & -0.19 & $-7.67^{\star *}$ & $a a$ \\
\hline-0.26 & 0.03 & 0.33 & -0.32 & $6.07^{\star \star}$ & $a d$ \\
\hline$-7.48^{* *}$ & $4.17^{\star *}$ & $2.67^{* *}$ & 0.81 & $17.27^{* *}$ & $d d$ \\
\hline \multicolumn{6}{|c|}{ Cross $5(2 \times 3)$} \\
\hline 89.93 & 16.10 & 9.33 & 1.02 & 66.07 & $m$ \\
\hline $1.21^{*}$ & 0.30 & 0.00 & -0.25 & -4.43 & $a$ \\
\hline $2.92^{* *}$ & $-11.84^{* *}$ & $1.92^{*}$ & 0.30 & $3.75^{\star *}$ & $d$ \\
\hline $5.95^{\star *}$ & $-12.46^{\star *}$ & $1.33^{*}$ & 0.44 & 1.53 & aa \\
\hline $1.72^{*}$ & 0.42 & 0.08 & -0.21 & -3.65 & $a d$ \\
\hline$-7.37^{* *}$ & $19.62^{* *}$ & $-1.17^{\star}$ & -0.93 & $-13.37^{\star \star}$ & $d d$ \\
\hline
\end{tabular}

${ }^{*},{ }^{* *}$ Significant and highly significant at the 0.05 and 0.01 level of probability, respectively. ( $m, a, d, a a, a d$ and $d d=$ population mean, additive, dominant, additive $\mathrm{x}$ additive, additive $\mathrm{x}$ dominant and dominant $x$ dominant gene action, respectively).

To be Contd. 
Table (8) Cont'

\begin{tabular}{|c|c|c|c|c|c|}
\hline $\begin{array}{c}\text { Moisture } \\
\text { content (\%) }\end{array}$ & T.S.S (\%) & $\begin{array}{c}\text { Fruit } \\
\text { netting } \\
\text { degree }\end{array}$ & $\begin{array}{l}\text { Fruit } \\
\text { shape } \\
\text { index }\end{array}$ & $\begin{array}{c}\text { Fruit flesh } \\
\text { thickness } \\
(\%)\end{array}$ & Gene action \\
\hline \multicolumn{6}{|c|}{ Cross $6(2 \times 4)$} \\
\hline 89.53 & 15.28 & 10.00 & 1.03 & 67.13 & $m$ \\
\hline-1.35 & 2.55 & 2.00 & 0.15 & $1.50^{* *}$ & $a$ \\
\hline$-7.81^{* *}$ & -3.13 & -7.00 & -0.21 & $-14.95^{\star *}$ & $d$ \\
\hline $9.97^{*}$ & -4.96 & -7.33 & -0.28 & $-20.86^{\star \star}$ & $a a$ \\
\hline-1.95 & 2.39 & 2.33 & 0.11 & $1.72^{* *}$ & $a d$ \\
\hline$-47.84^{\star *}$ & $9.18^{* *}$ & $14.00^{*}$ & 0.61 & $32.30^{* *}$ & $d d$ \\
\hline \multicolumn{6}{|c|}{ Cross $7(2 \times 5)$} \\
\hline 91.64 & 13.5 & 9.83 & 1.03 & $68.13^{*}$ & $m$ \\
\hline 2.00 & 0.98 & 0.17 & -0.16 & $-5.40^{* *}$ & $a$ \\
\hline$-3.00^{\star *}$ & $-1.73^{\star \star}$ & -0.76 & -0.13 & $-11.23^{\star *}$ & $d$ \\
\hline$-2.97^{\star *}$ & $-0.76^{\star *}$ & -1.00 & -0.08 & $-15.20^{\star *}$ & $a a$ \\
\hline-2.85 & 1.15 & 0.17 & -0.22 & $-2.63^{* *}$ & $a d$ \\
\hline $2.28^{* *}$ & $0.63^{\star *}$ & $0.67^{*}$ & -0.09 & $23.27^{\star \star}$ & $d d$ \\
\hline \multicolumn{6}{|c|}{ Cross $8(3 \times 4)$} \\
\hline $90.54^{*}$ & 13.92 & 9.83 & 0.96 & 66.20 & $m$ \\
\hline-0.15 & 0.02 & -0.83 & 0.01 & $1.57^{* \star}$ & $a$ \\
\hline$-0.92^{* *}$ & $8.45^{\star *}$ & -1.34 & -0.04 & $-12.10^{\star *}$ & $d$ \\
\hline $0.18^{\star *}$ & $7.03^{\star *}$ & -3.00 & -0.06 & $-12.46^{\star *}$ & aa \\
\hline-1.26 & -0.30 & 0.82 & -0.06 & $1.00^{* *}$ & $a d$ \\
\hline $1.76^{* *}$ & $-9.70^{* *}$ & $3.35^{*}$ & 0.48 & $11.27^{* *}$ & $d d$ \\
\hline \multicolumn{6}{|c|}{ Cross $9(3 \times 5)$} \\
\hline 90.14 & 15.62 & 9.00 & 1.06 & 61.20 & $m$ \\
\hline 1.62 & 0.12 & -0.17 & 0.01 & -1.33 & $a$ \\
\hline$-0.07^{\star \star}$ & $-4.34^{*}$ & 4.25 & -0.27 & $20.95^{\star \star}$ & $d$ \\
\hline$-0.54^{\star *}$ & $-3.76^{*}$ & 3.66 & 0.26 & $16.13^{\star \star}$ & $a a$ \\
\hline 0.21 & 0.16 & -0.25 & -0.09 & 0.65 & $a d$ \\
\hline $6.49^{* *}$ & 2.42 & -4.50 & 0.36 & -6.83 & $d d$ \\
\hline \multicolumn{6}{|c|}{ Cross $10(4 \times 5)$} \\
\hline 91.53 & 14.60 & 9.83 & 1.15 & $62.07^{*}$ & $m$ \\
\hline 2.22 & -0.90 & 1.00 & 0.13 & $-0.40^{* *}$ & $a$ \\
\hline $0.90^{* *}$ & $1.64^{*}$ & -2.50 & -0.47 & $1.78^{\star \star}$ & $d$ \\
\hline$-1.21^{\star *}$ & $0.47^{\star}$ & -2.67 & -0.45 & $7.07^{\star \star}$ & aa \\
\hline 1.90 & -0.58 & 0.67 & 0.10 & $2.15^{\star \star}$ & $a d$ \\
\hline $1.32^{\star *}$ & $0.38^{\star *}$ & $5.00^{\star *}$ & 0.06 & $-8.63^{\star *}$ & $d d$ \\
\hline
\end{tabular}

*, ** Significant and highly significant at the 0.05 and 0.01 level of probability, respectively ( $m, a, d, a a, a d$ and $d d=$ population mean, additive, dominant, additive $\mathrm{x}$ additive, additive $\mathrm{x}$ dominant and dominant $x$ dominant gene action, respectively).

\section{Correlation coefficient and path analysis}

Correlation coefficient values are presented in Table (9). Values of the correlation coefficient were positive and significant or highly significant among the following characters: plant length with each of average fruit weight / plant and total yield / plant. Flowering date with maturity (days). Maturity (days) with the netting degree. Average fruit number with each of total yield /plant and netting degree. Average fruit weight with each of total yield / plant, fruit shape index. Total yield / plant with each of netting degree, TSS. TSS with moisture 
content. Negative and significant or highly significant correlation values were found among maturity date with of No. of branches per plant.

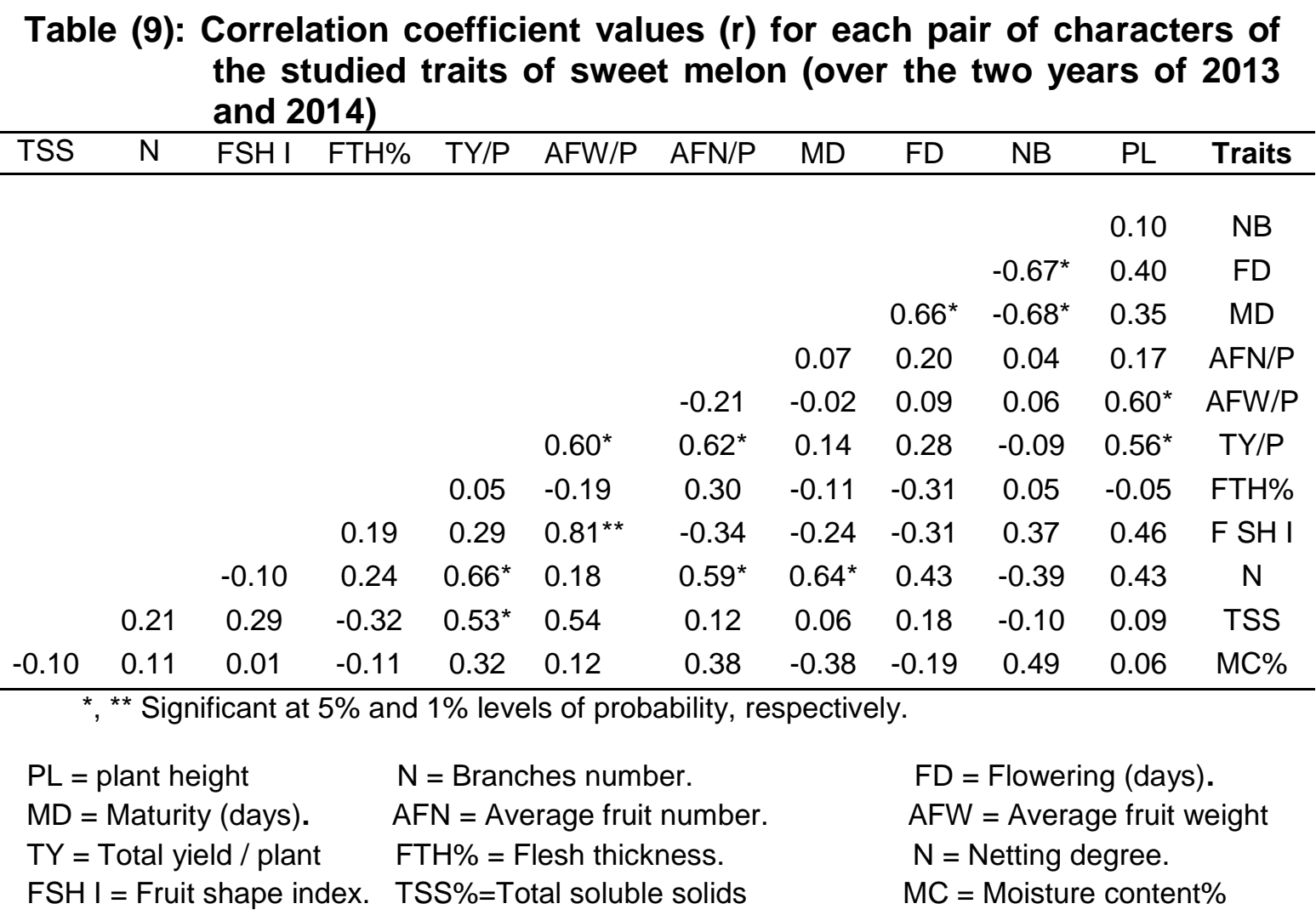

It could be concluded from results, listed in Table (9), that the characters plant length, number branches / plant and average fruit weight were good determinates of total yield / plant. These results suggesting that selection should be practiced for high yield production based on the plants which recorded highest values respecting to these three characters. Similar trend, more or less, of these results were found by Ibrahim and Ramadan (2013) who found highly significant positive correlation among total yield / plant with each of plant length and average fruit weight. The results of Wahba (2004) on some local cultivars and hybrids of sweet melon showed a positive correlation between flesh thickness and fruit diameter and among fruit weight and each of fruit length, fruit diameter and seed cavity diameter. The negative association was detected between flesh thickness and seed cavity / fruit diameter.

The Direct and indirect effects of average fruit number, average fruit weight $(\mathrm{kg})$, plant length $(\mathrm{cm})$, branches number and fruit shape index on total yield / plant $(\mathrm{kg})$ were tabulated in Table (10). It could be noted from the data of path coefficient analysis that the direct effect was positive and highly in magnitude for fruit number per plant, plant length and fruit shape index. So, selection for these characters may be effective in improving the total yield per plant $(\mathrm{kg})$. The Indirect effect was highly positive for the average fruit weight through its relation with fruit flesh thickness $(0.5761)$, So, selection for high fruit flesh thickness may be effective in improving total yield through its relation with average fruit weight. Similar results were found by Feyzian et al. (2009) and Abou kamer (2011) on melon, they found direct positive effect for average fruit 
number on total yield / plant and indirect positive effect for branches number through its relation with average fruit number / plant on total yield.

Table (10): Direct and indirect effects of some characters (Average No. of fruit per plant, average fruit weight per plant, plant length, No. of branches per plant and fruit flesh thickness) on total yield per plant

\begin{tabular}{ccccccc}
\hline Total effect & $\mathrm{FTH}$ & $\mathrm{BN}$ & $\mathrm{PL}$ & $\mathrm{FW}$ & $\mathrm{FN}$ & traits \\
\hline 0.6200 & -0.2418 & -0.0082 & 0.1000 & 0.0341 & $\underline{0.7359}$ & $\mathrm{FN}$ \\
0.6000 & 0.5761 & -0.0122 & 0.3529 & $\underline{-0.1622}$ & -0.1545 & $\mathrm{FW}$ \\
0.5600 & -0.0356 & -0.0204 & $\underline{0.5882}$ & -0.0973 & 0.1251 & $\mathrm{PL}$ \\
0.0900 & 0.0356 & $\underline{-0.2041}$ & 0.0588 & -0.0097 & 0.0294 & $\mathrm{BN}$ \\
0.2900 & $\underline{0.7112}$ & -0.0102 & -0.294 & -0.1314 & -0.2502 & $\mathrm{FTH}$ \\
\hline
\end{tabular}

R.E $=0.2950405$

$\mathrm{AFN}=$ Average fruit number.

$\mathrm{AFW}=$ Average fruit weight $(\mathrm{kg})$.

$\mathrm{PL}=$ plant height.

$\mathrm{BN}=$ Branches number.

$\mathrm{FTH} \%=$ Flesh thickness.

\section{REFERENCES}

Abou kamer, M. E. (2011). Genetical studies on some important characters on melon (Cucumis melo L.). A Thesis submitted, Alex. Univ. Egypt.

Abu Arak, S.A. (2013). Genetic variation in growth and yield of crosses between Snake melon (Cucumis melo var. conomon) and Cantaloupe (Cucumis melo L. var. recticularis) Lambert Acad. Publishing proj. Code Sut. 3-303- 53-12-29.

Allard, R.W.(1960). Principles of plant breeding .London, John Wiley and Sons, pp.458.

Anne K. A., N. S. Glauber, Q. A. Manoel, P. L. Elaíne and F. C. José (2011). Diallel analysis of yield and quality traits of melon fruits. Crop Breed. \& Appl. Biotech., 11: 313-319.

Chamnan, I. P. (2006). Heritability, Heterosis and Correlations of Fruit Characters and Yield in Thai Slicing Melon (Cucumis melo L. var. conomon Makino). Kasetsart J. (Nat. Sci.), 40: $20-25$.

Dospekove, B.A. (1984). Field experimental, statistical procedures. Mir Publishers: 349.

Fernaindez S., E. Moreno, I. Eduardo J.M. Alvarez and A.J. Monforte (2009). On the genetic control of heterosis for fruit shape in melon (Cucumis melo L.). J. Hered., 100 (2): 229-235.

Feyzian, E., H. Dehghani, A. M. Rezai and M. J. Javaran (2009). Diallel cross analysis for maturity and yield - related traits in melon (Cucumis melo L.) Euphytica, 168: 215-223.

Gamble, E.E. (1962). Gene effects in corn (Zea mays L.) I. Separation and relative importance of gene effects for yield. Canad. J. Plant Sci., 42:339-348.

Goldman, A. (2002). Melons for the passionate Grower, Artisan, New York.

Hayman, B.I. (1958). The separation of epistatic from additive and dominance variation in generation means. Heredity, 12:371-390. 
Ibrahim E.A and A.Y. Ramadan (2013). Correlation and Path Coefficient analysis in sweet melon (Cucumis melo var aegyptiacus L.) under Irrigation and Drought conditions. Pakistan J. of Biol. Sci., 16: 610- 616.

Kirkbride, J.H. (1993). Biosystematic monograph of the genus Cucumis (Cucurbitaceae). Parkway Publishers, Boone (NC, USA), 159 pp.

Pornsuriya, P. (2009). Study on genetic effect in fruit shape of Oriental Pickling melon. J. Agric. Technology, 5(2):385-390.

Reddy .B.P.K., H. Begum, N. Sunil, M.T. Reddy(2013). Variance component analysis of quantitative traits in muskmelon (Cucumis melo L.). Trakia J. Sci., 2: 118-128.

Wahba, M. (2004). The use of certain genetic parameters in improving some vegetables crops. Alex. Sci. Excel., 25(3):457-464.

Zalapa, J.E., J.E. Stab and J.D. Creight (2006). Generation means analysis of plant architectural trait and fruit yield in melon plant breeding. J. Compilation, 125: 482-487.

$$
\text { الملخص العربي }
$$

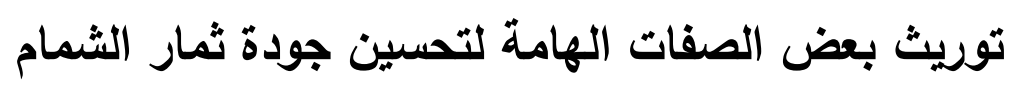

$$
\begin{aligned}
& \text { "محمد عيسي عبد الرحمن أبو قمر , "منى محمد يسرى جابر , "أحمد قطب قطب حاتم }
\end{aligned}
$$

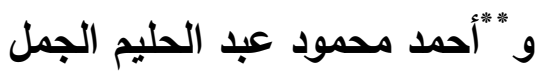

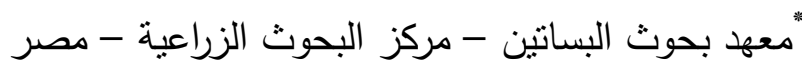

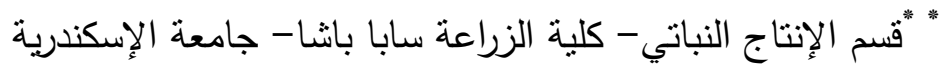

أجريت هذه الدراسة خلال ثلاثة أعوام متتالية 2012, 2013 و و2014 , حيث تم تنفيذ التجربة في محطة

بحوث البساتين-الصبحية -الإنكندرية بالإضافة لمنطقة فوه - محافظة كفر الثيخ- جمهورية مصر العربية,

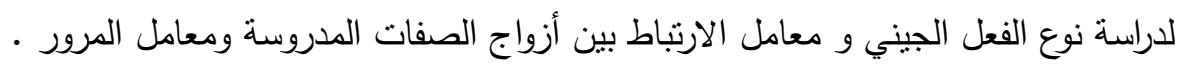

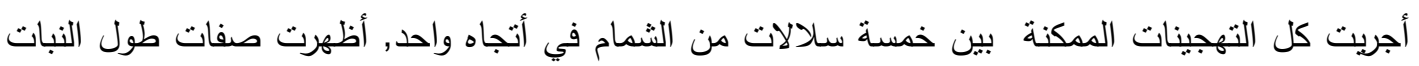

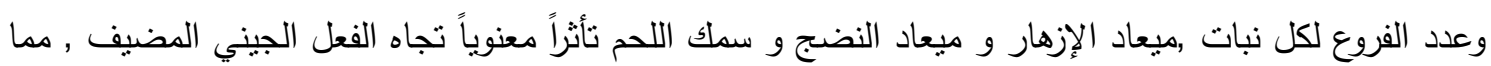

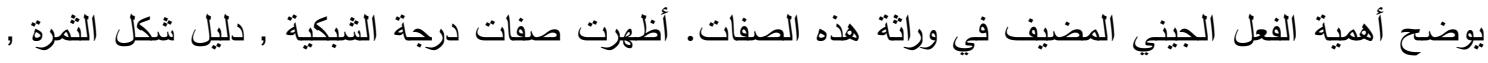

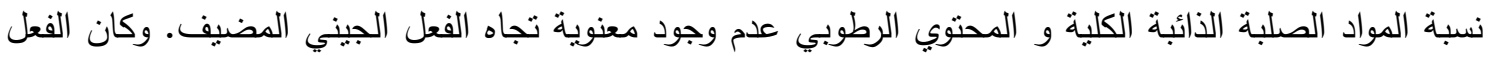

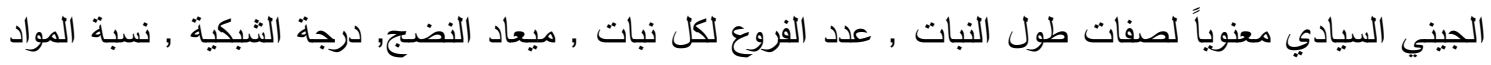

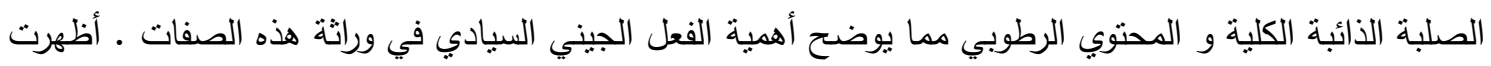

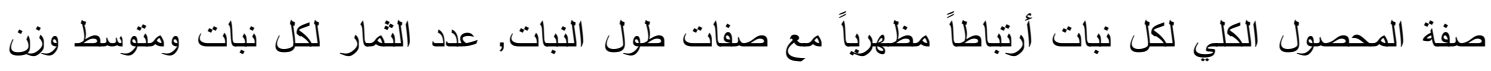

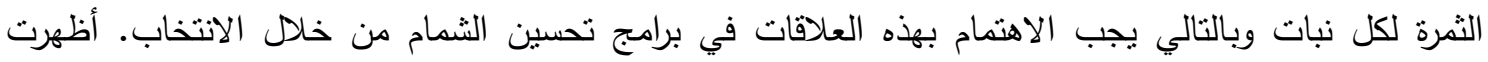

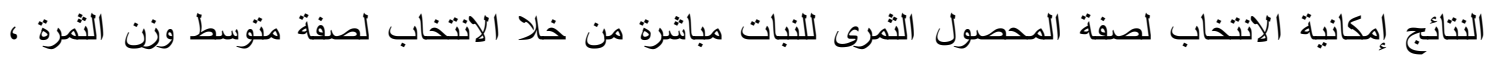
وأيضا يمكن الانتخاب لصفة المحصول الثمرى بطريقة غير مباثرة من خلال الانتخاب لصفة سمك لحم الثمرة الثرة

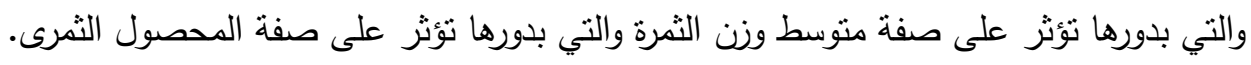

